\title{
VOTER MODEL ON SIGNED SOCIAL NETWORKS
}

\author{
Yanhua Li, ${ }^{1, *}$ Wei Chen, ${ }^{2}$ Yajun Wang, ${ }^{2}$ and Zhi-Li Zhang ${ }^{1}$ \\ ${ }^{1}$ Department of Computer Science \& Engineering, University of Minnesota, \\ Minneapolis, Minnesota, USA \\ ${ }^{2}$ Microsoft Research
}

\begin{abstract}
Online social networks (OSNs) are becoming increasingly popular and are generating great interest in the study of the influence diffusion and influence maximization with applications to online viral marketing. Existing studies focus on social networks with only friendship relations, whereas the foe or enemy relations that commonly exist in many OSNs, e.g., Epinions and Slashdot, are completely ignored. In this study, we make the first attempt to investigate the influence diffusion and influence maximization in OSNs with both friend and foe relations, which are modeled using positive and negative edges on signed networks. In particular, we extend the classic voter model to signed networks and analyze the dynamics of influence diffusion of two opposite opinions. We first provide systematic characterization of both short-term and long-term dynamics of influence diffusion in this model and illustrate that the steady state behaviors of the dynamics depend on three types of graph structures, which we refer to as balanced graphs, anti balanced graphs, and strictly unbalanced graphs. We then apply our results to solve the influence maximization problem and develop efficient algorithms to select initial seeds of one opinion that maximize either its short-term influence coverage or long-term steady state influence coverage. Extensive simulation results on both synthetic and real-world networks, such as Epinions and Slashdot, confirm our theoretical analysis on influence diffusion dynamics, and demonstrate the efficacy of our influence maximization algorithm over other heuristic algorithms.
\end{abstract}

\section{INTRODUCTION}

As the popularity of online social networks (OSNs) such as Facebook and Twitter continuously increases, OSNs have become an important platform for the dissemination of news, ideas, opinions, etc. The openness of the OSN platforms and the richness of contents and user interaction information enable intelligent online recommendation systems and viral marketing techniques. For example, if a company wants to promote a new product, it may identify a set of influential users in the online social network and provide them with free sample products. They hope that these influential users could influence their friends and friends of friends in the network and so on, generating a large influence cascade so that many users adopt their product as a result of such word-of-mouth promotion. The question is how to select the initial users, given a limited budget on free samples, in order to influence the largest number of people to purchase the product through this "word-of-mouth"

*A preliminary version of the results in this paper appeared in [27].

Address correspondence to Yanhua Li, Department of Computer Science \& Engineering, University of Minnesota, Minneapolis, MN 55414, USA. E-mail: yanhua@cs.umn.edu

Color versions of one or more of the figures in the article can be found online at www.tandfonline.com/uinm. 
process. Similar situations could apply to the promotion of ideas and opinions, such as political candidates trying to find early supporters for their political proposals and agendas, and government authorities or companies trying to win public support by finding and convincing an initial set of early adopters of their ideas.

The above problem is referred to as the influence maximization problem in the literature, which has been extensively studied in recent years $[7-9,16-18,21,22,26,37,38]$. In these studies, several influence diffusion models are proposed to formulate the underlying influence propagation processes, including the linear threshold (LT) model, the independent cascade (IC) model, the voter model, etc. A number of approximation algorithms and scalable heuristics are designed under these models to solve the influence maximization problem.

However, all existing studies look only at networks with positive (i.e., friend, altruism, or trust) relationships, whereas in reality, relationships also include negative ones, such as foe, spite, or distrust relationships. On ebay, users develop trust and distrust in agents in the network; in online review and news forums, such as Epinions and Slashdot, readers approve or denounce reviews and articles of each other. Some recent studies [10, 24, 25] already look into the network structures with both positive and negative relationships. As a common sense exploited in many existing social influence studies [7-9, 16, 21], positive relationships carry the influence in a positive manner, i.e., you would more likely trust and adopt your friends' opinions. In contrast, we consider that negative relationships often carry influence in a reverse direction - if your foe chooses one opinion or votes for one candidate, you would more likely be influenced to do the opposite. This echoes the principles that "the friend of my enemy is my enemy" and "the enemy of my enemy is my friend." Structural balance theory has been developed based on these assumptions in social science (see Chapter 5 of [14] and the references therein). We acknowledge that in real social networks, people's reactions to the influence from their friends or foes could be complicated: i.e., one could take the opposite opinion of what a foe suggests for one situation or topic, but may adopt the suggestion from the same person for a different topic, because the foe's expertise in that particular topic is trusted. In this study, we consider the influence diffusion for a single topic in which one always takes the opposite opinion of what a foe suggests. This is our first attempt to model influence diffusion in signed networks, and such topicdependent simplification is commonly employed in prior influence diffusion studies on unsigned networks [7-9, 16, 18,21]. Our work aims at providing a mathematical analysis of the influence diffusion dynamic incorporated with negative relationship and applying the analysis to the algorithmic problem of influence maximization.

\subsection{Our Contributions}

In this work, we extend the classic voter model $[13,20]$ to incorporate negative relationships for modeling the diffusion of opinions in a social network. Given an unsigned directed graph (digraph), the basic voter model works as follows. At each step, every node in the graph randomly picks one of its outgoing neighbors and adopts the opinion of this neighbor. Thus, the voter model is suitable to interpret and model opinion diffusions where people's opinions may switch back and forth based on their interactions with other people in the network. To incorporate negative relationships, we consider signed digraphs in which every directed edge is either positive or negative, and we consider the diffusion of two opposite opinions, e.g., black and white colors. We extend the voter model to signed digraphs such that at each step, every node randomly picks one of its outgoing neighbors; 
and if the edge to this neighbor is positive, the node adopts the neighbor's opinion, but if the edge is negative, the node adopts the opposite of the neighbor's opinion (Section 2).

We provide detailed mathematical analysis on the voter model dynamics for signed networks (Section 3). For short-term dynamics, we derive the exact formula for opinion distribution at each step. For long-term dynamics, we provide closed-form formulas for the steady state distribution of opinions. We show that the steady state distribution depends on the graph structure: we divide signed digraphs into three classes of graph structuresbalanced graphs, antibalanced graphs, and strictly unbalanced graphs; each of which leads to a different type of steady state distribution of opinions. Although balanced and unbalanced graphs have been extensively studied by structural balance theory in social science [14], the antibalanced graphs form a new class that has not been covered before, to the best of our knowledge. Moreover, our long-term dynamics cover not only strongly connected and aperiodic digraphs that most of such studies focus on, but also weakly connected and disconnected digraphs, making our study more comprehensive.

We then study the influence maximization problem under the voter model for signed digraphs (Section 4). The problem here is to select, at most, $k$ initial white nodes while all others are black, so that either over the short term or long term the expected number of white nodes is maximized. This corresponds to the scenario in which one opinion dominates the public and an alternative opinion (e.g., a competing political agenda, or a new innovation) tries to win over supporters as much as possible by selecting some initial seeds to influence. We provide efficient algorithms that find optimal solutions for both short-term and longterm cases. In particular, for long-term influence maximization, our algorithm provides a comprehensive solution covering weakly connected and disconnected signed digraphs, with nontrivial computations on influence coverage of seed nodes.

Finally, we conduct extensive simulations on both real-world and synthetic networks to verify our analysis and to show the effectiveness of our influence maximization algorithm (Section 5). The simulation results demonstrate that our influence maximization algorithms perform much better than other heuristic algorithms.

To the best of our knowledge, we are the first to study influence diffusion and influence maximization in signed networks and the first to apply the voter model to this case and provide efficient algorithms for influence maximization under voter model for signed networks.

\subsection{Related Work}

In this subsection, we discuss the topics that are closely related to our problem, such as: (1) influence maximization and voter model, (2) signed networks, and (3) competitive influence diffusion.

1.2.1. Influence Maximization and Voter Model. Influence maximization has been extensively studied in the literature. The initial work [21] proposes several influence diffusion models and provides the greedy approximation algorithm for influence maximization. More recent works [7-9, 16, 18, 22, 26,37] study efficient optimizations and scalable heuristics for the influence maximization problem. In particular, the voter model is proposed in $[13,20]$ and is suitable for modeling opinion diffusions in which people may switch opinions back and forth from time to time because of the interactions with other people in the network. Even-Dar and Shapira [16] study the influence maximization problem in the voter model on simple unsigned and undirected graphs, and they show that the best seeds for long-term influence maximization are simply the highest degree nodes. As a contrast, 
we show in this article that seed selections for signed digraphs are more sophisticated, especially for weakly connected or disconnected signed digraphs. Chung and Tsiatas [12] consider the voter models on hypergraphs as random walks on the associated weighted directed state graphs with various restrictions, such as "memoryless" or "partial memoryless" random walks, and provide bounds of the convergence time in various voter model settings. More voter-model-related research is conducted in physics domain, where the voter model, the zero-temperature Glauber dynamics for the Ising model, invasion process, and other related models of population dynamics belong to the class of models with two absorbing states and epidemic spreading dynamics $[1,36,40]$. However, none of these works studies the influence diffusion and influence maximization of the voter model under signed networks.

1.2.2. Signed Networks. The signed networks with both positive and negative links have gained attention recently [3,23-25]. In [24, 25], the authors empirically study the structure of real-world social networks with negative relationships based on two social science theories: balance theory and status theory. Kunegis et al. [23] study the spectral properties of the signed undirected graphs, with applications in link predictions, spectral clustering, etc. Borgs et al. [3] propose a generalized PageRank algorithm for signed networks with application to online recommendations, where the distrust relations are considered as adversarial or arbitrary user behaviors; thus, the outgoing relations of distrusted users are ignored while ranking nodes. Our algorithm can also be considered an influenceranking algorithm that generalizes the PageRank algorithm, but we treat distrust links as generating negative influence rather than ignoring distrusted users' opinions, and thus our ranking method is different from [3]. None of the above studies influence diffusion and influence maximization in signed networks.

1.2.3. Competitive Influence Diffusion. A number of recent studies focus on competitive influence diffusion and maximization [2,4-6, 19,35], in which two or more competitive opinions or innovations are diffusing in the network. Although they consider two or more competitive or opposing influence diffusions, they are all on unsigned networks, differing from our present study on diffusion with both positive and negative relationships.

\section{VOTER MODEL FOR SIGNED NETWORKS}

We consider a weighted directed graph (digraph) $G=(V, E, A)$, where $V$ is the set of vertices, $E$ is the set of directed edges, and $A$ is the weighted adjacency matrix with $A_{i j} \neq 0$ if and only if $(i, j) \in E$, with $A_{i j}$ as the weight of edge $(i, j)$. The voter model was first introduced for unsigned graphs, with nonnegative adjacency matrices $A$ 's. In this model, each node holds one of two opposite opinions, represented by black and white. Initially, each node has either black or white. At each step $t \geq 1$, every node $i$ randomly picks one outgoing neighbor $j$ with the probability proportional to the weight of $(i, j)$, namely $A_{i j} / \sum_{\ell} A_{i \ell}$, and changes its color to $j$ 's color. The voter model also has a random walk interpretation. If a random walk starts from $i$ and stops at node $j$ at step $t$, then $i$ 's color at step $t$ is $j$ 's color at step 0 .

In this study, we extend the voter model to signed digraphs, in which the adjacency matrix $A$ may contain negative entries. A positive entry $A_{i j}$ represents that $i$ considers $j$ as a friend or $i$ trusts $j$, and a negative $A_{i j}$ means that $i$ considers $j$ as a foe or $i$ distrusts $j$. The absolute value $\left|A_{i j}\right|$ represents the strength of this trust or distrust relationship. The voter model is thus extended naturally such that one always takes the same opinion from a friend, and the opposite opinion of a foe. Technically, at each step $t \geq 1, i$ randomly picks one outgoing neighbor $j$ with probability $\left|A_{i j}\right| / \sum_{\ell}\left|A_{i \ell}\right|$, and if $A_{i j}>0$ (or edge $(i, j)$ is 


\begin{tabular}{|c|c|}
\hline $\begin{array}{l}G=(V, E, A) \\
\bar{G}=(V, E, \bar{A})\end{array}$ & $\begin{array}{l}G \text { is a signed digraph, with signed adjacency matrix } A \text { and } \bar{G} \\
\text { is the unsigned version of } G \text {, with adjacency matrix } \bar{A}\end{array}$ \\
\hline$A^{+}, A^{-}$ & $\begin{array}{l}A^{+}\left(\text {resp. } A^{-}\right) \text {is the non-negative adjacency matrix represent- } \\
\text { ing positive (resp. negative) edges of } G \text {, with } A=A^{+}-A^{-} \\
\text {and } \bar{A}=A^{+}+A^{-} \text {. }\end{array}$ \\
\hline $\begin{array}{l}\mathbf{1}, \pi, x_{0}, x_{t}, x, \\
x_{e}, x_{o}\end{array}$ & $\begin{array}{l}\text { Vector forms. All vectors are }|V| \text {-dimensional column vectors } \\
\text { by default; } \mathbf{1} \text { is all one vector, } \pi \text { is the stationary distribution } \\
\text { of the ergodic digraph } \bar{G} ; x_{0} \text { (resp. } x_{t} \text { ) is the white color } \\
\text { distribution at the beginning (resp. at step } t) ; x \text { is the steady } \\
\text { state white color distribution; } x_{e} \text { (resp. } x_{o} \text { ) is the steady state } \\
\text { white color distribution for even (resp. odd) steps. }\end{array}$ \\
\hline$d, d^{+}, d^{-}, D$ & $\begin{array}{l}d, d^{+}, \text {and } d^{-} \text {are weighted out-degree vectors of } G, \text { where } \\
d=\bar{A} \mathbf{1}, d^{+}=A^{+} \mathbf{1} \text {, and } d^{-}=A^{-} \mathbf{1} ; D=\operatorname{diag}[d] \text { is the } \\
\text { diagonal degree matrix filled with entries of } d \text {. }\end{array}$ \\
\hline$P, \bar{P}$ & $\begin{array}{l}P=D^{-1} A \text { is the signed transition matrix of } G \text { and } \bar{P}= \\
D^{-1} \bar{A} \text { is the transition probability matrix of } \bar{G} .\end{array}$ \\
\hline$v_{Z}, \hat{v}_{S}, \hat{v}_{Z, S_{Z}}$ & $\begin{array}{l}\text { Given a vector } v \text {, a node set } Z \subseteq V, v_{Z} \text { is the projection of } \\
v \text { on } Z \text {. Given a partition } S, \bar{S} \text { of } V, \hat{v}_{S} \text { is signed such that } \\
\hat{v}_{S}(i)=v(i) \text { if } i \in S \text {, and } \hat{v}_{S}(i)=-v(i) \text { if } i \notin S \text {. Given a } \\
\text { partition } S_{Z}, \bar{S}_{Z} \text { of } Z, \hat{v}_{Z, S_{Z}} \text { is taking the projection of } v \text { on } Z \\
\text { first, then negating the signs for entries in } \bar{S}_{Z} \text {. }\end{array}$ \\
\hline$I, \hat{I}_{S}, B_{Z}$ & $\begin{array}{l}I \text { is the identity matrix. } \hat{I}_{S}=\operatorname{diag}\left[\hat{\mathbf{1}}_{S}\right] \text { is the signed identity } \\
\text { matrix. } B_{Z} \text { is the projection of a matrix } B \text { to } Z \subseteq V \text {. }\end{array}$ \\
\hline
\end{tabular}

Table I Notations and terminologies.

positive) then $i$ changes its color to $j$ 's color, but if $A_{i j}<0$ (or edge $(i, j)$ is negative) then $i$ changes its color to the opposite of $j$ 's color. The random-walk interpretation can also be extended for signed networks: if the $t$-step random walk from $i$ to $j$ passes an even number of negative edges, then $i$ 's color at step $t$ is the same as $j$ 's color at step 0 ; whereas if it passes an odd number of negative edges, then $i$ 's color at step $t$ is the opposite of $j$ 's color at step 0 .

Given a signed digraph $G=(V, E, A)$, let $G^{+}=\left(V, E^{+}, A^{+}\right)$and $G^{-}=$ $\left(V, E^{-}, A^{-}\right)$denote the unsigned subgraphs consisting of all positive edges $E^{+}$and all negative edges $E^{-}$, respectively, where $A^{+}$and $A^{-}$are the corresponding nonnegative adjacency matrices. Thus, we have $A=A^{+}-A^{-}$. Similar to unsigned digraphs, $G$ is aperiodic if the greatest common divisor of the lengths of all cycles in $G$ is 1 , and $G$ is ergodic if it is strongly connected and aperiodic. A sink component of a signed digraph is a strongly connected component that has no outgoing edges to any nodes outside the component. When studying the long-term dynamics of the voter model, we assume that all signed, strongly connected components are ergodic. We first study the case of ergodic graphs, and then extend it to the more general case of weakly connected or disconnected graphs with ergodic sink components. Table I provides notations and terminologies used in this article. Note one basic fact that we often use in studying long-term convergence behavior: if matrix $P$ satisfies $\lim _{t \rightarrow \infty} P^{t}=\mathbf{0}$, then $I-P$ is invertible and $(I-P)^{-1}=\lim _{t \rightarrow \infty} \sum_{i=0}^{t} P^{i}$.

\section{ANALYSIS OF VOTER MODEL DYNAMICS ON SIGNED DIGRAPHS}

In this section, we study the short-term and long-term dynamics of the voter model on signed digraphs. In particular, we answer the following two questions. 
1. Short-term dynamics: Given an initial distribution of black and white nodes, what is the distribution of black and white nodes at step $t>0$ ?

2. Convergence of voter model: Given an initial distribution of black and white nodes, would the distribution converge, and what is the steady state distribution of black and white nodes?

\subsection{Short-Term Dynamics}

To study voter model dynamics on signed digraphs, we first define the signed transition matrix as follows.

Definition 3.1. (Signed transition matrix) Given a signed digraph $G=(V, E, A)$, we define the signed transition matrix of $G$ as $P=D^{-1} A$, where $D=\operatorname{diag}\left[d_{i}\right]$ is the diagonal matrix and $d_{i}=\sum_{j \in V}\left|A_{i j}\right|$ is the weighted out-degree of node $i$.

The next proposition characterizes the dynamics of the voter model at each step, using the signed transition matrix.

Proposition 3.2. Let $G=(V, E, A)$ be a signed digraph and denote the initial white color distribution vector as $x_{0}$, i.e., $x_{0}(i)$ represents the probability that node $i$ is white initially. Then, the white color distribution at step $t$, denoted by $x_{t}$ can be computed as

$$
x_{t}=P^{t} x_{0}+\left(\sum_{i=0}^{t-1} P^{i}\right) g^{-}
$$

where $g^{-}=D^{-1} A^{-} \mathbf{1}$, i.e., $g^{-}(i)$ is the weighted fraction of outgoing negative edges of node $i$.

Proof. Based on the signed digraph voter model defined in Section 2, $x_{t}$ can be iteratively computed as

$$
x_{t}(i)=\sum_{j \in V} \frac{A_{i j}^{+}}{d_{i}} x_{t-1}(j)+\sum_{j \in V} \frac{A_{i j}^{-}}{d_{i}}\left(1-x_{t-1}(j)\right) .
$$

In matrix form, we have

$$
x_{t}=D^{-1} A x_{t-1}+D^{-1} A^{-} \mathbf{1}=P x_{t-1}+g^{-},
$$

which yields (3.1) by repeatedly applying (3.3).

\subsection{Convergence of Signed Transition Matrix with Relation to Structural Balance of Signed Digraphs}

Equation (3.1) infers that the long-term dynamics, i.e., the vector $x_{t}$ when $t$ goes to infinity, depends critically on the limit of $P^{t}$ and $\sum_{i=0}^{t-1} P^{i}$. In the following, we show that the limiting behavior of the two matrix sequences is fundamentally determined by the structural balance of signed digraph $G$, which connects to the social balance theory 
well studied in the social science literature (cf. [14]). We now define three types of signed digraphs based on their balance structures.

Definition 3.3. (Structural balance of signed digraphs) Let $G=(V, E, A)$ be a signed digraph.

1. Balanced digraph. $G$ is balanced if there exists a partition $S, \bar{S}$ of nodes in $V$, such that all edges within $S$ and $\bar{S}$ are positive and all edges across $S$ and $\bar{S}$ are negative.

2. Antibalanced digraph. $G$ is antibalanced if there exists a partition $S, \bar{S}$ of nodes in $V$, such that all edges within $S$ and $\bar{S}$ are negative and all edges across $S$ and $\bar{S}$ are positive.

3. Strictly unbalanced digraph. $G$ is strictly unbalanced if $G$ is neither balanced nor antibalanced.

The balanced digraphs defined previously correspond to the balanced graphs originally defined in social balance theory. It is known that a balanced graph can be equivalently defined by the condition that all circles in $G$ without considering edge directions, contain an even number of negative edges [14]. Conversely the concept of antibalanced digraphs seems not to appear in the social balance theory. Note that balanced digraphs and antibalanced digraphs are not mutually exclusive. For example, a fournode circle with one pair of nonadjacent edges being positive and the other pair being negative is both balanced and antibalanced. However, for studying long-term dynamics, we need the above categorization only for aperiodic digraphs, for which we show below that balanced digraphs and antibalanced digraphs are mutually exclusive.

Proposition 3.4. An aperiodic digraph $G$ cannot be both balanced and antibalanced.

Proof. Suppose, for a contradiction, that an aperiodic digraph $G$ is both balanced and antibalanced. By the equivalent condition of balanced graphs, we know that all cycles of $G$ have an even number of negative edges. Because an antibalanced graph will become balanced if we negate the signs of all its edges, we know that all cycles of $G$ also have an even number of positive edges. Therefore, all cycles of $G$ must have an even number of edges, which means their lengths have a common divisor 2, contradicting to the assumption that $G$ is aperiodic.

With the above proposition, we know that balanced graphs, antibalanced graphs, and strictly unbalanced graphs indeed form a classification of aperiodic digraphs, where antibalanced graphs and strictly unbalanced graphs together correspond to unbalanced graphs in the social balance theory. We identify antibalanced graphs as a special category because they have a unique long-term dynamic behavior different from other graphs. An example of an antibalanced graph is a graph with only negative edges.

3.2.1. Case of Ergodic Signed Digraphs. Now, we discuss the limiting behavior of $P^{t}$ of ergodic signed digraphs with three balance structures. A signed digraph $G=(V, E, A)$ is ergodic if and only if for any node $i$, there always exists a signed path to any other node in $G$ and the common divisor of all cycle path lengths of $i$ is 1 . Here, a signed path $R$ in a signed graph $G$ is a sequence of nodes with the edges being directed from each node to the following one, where the length of the path, denoted as $|R|$, is the total number of directed edges in $R$. The sign of a path is positive if there is an even number of negative 
edges along the path; otherwise, the sign of a path is negative. Following, we first introduce Proposition 3.5, presenting that the balance structures of ergodic signed digraphs can be interpreted and distinguished in terms of the path lengths and path signs in $G$. As a result, Lemma 3.6 introduces the various limiting behaviors of $P^{t}$ of ergodic signed digraphs with respect to three balance structures.

Proposition 3.5. Let $G=(V, E, A)$ be an ergodic strictly unbalanced digraph. There exist two nodes $i$ and $j$, and two directed paths from $i$ to $j$ with the same length but different signs.

Proof. Given the following three statements, we prove

$$
\text { Statement } 1 \Rightarrow \text { Statement } 2 \Rightarrow \text { Statement } 3 \text {, }
$$

which in turn proves this proposition, i.e.,

$$
\neg \text { Statement } 3 \Rightarrow \neg \text { Statement } 1 .
$$

We assume that $G$ is a signed ergodic digraph.

Statement 1 : For any two nodes $i$ and $j$, all paths from $i$ to $j$ with the same length have same signs.

Statement 2 : For any two nodes $i$ and $j$, all paths from $i$ to $j$ with even length have same signs.

Statement $3: G$ is either balanced or antibalanced.

1. Proof by contradiction for

$$
\text { Statement } 1 \Rightarrow \text { Statement } 2 \text {. }
$$

We assume that in $G$, there exist two paths of even length $R_{e 1}$ and $R_{e 2}$ from $i$ to $j$ with different signs. Since $G$ is ergodic, by Proposition A.1 in Appendix A, there must exist a path, denoted by $R_{o}$, from $j$ to $i$ with odd length (no matter what sign it carries). Denote the length of these three paths as $\left|R_{e 1}\right|,\left|R_{e 2}\right|$, and $\left|R_{o}\right|$, respectively.

Then, $R_{c 1}=R_{e 1}+R_{o}$ forms a cycle at node $i$ with odd length $\left|R_{e 1}\right|+\left|R_{o}\right|$ and $R_{c 2}=R_{e 2}+R_{o}$ forms another cycle at $i$ with odd length $\left|R_{e 2}\right|+\left|R_{o}\right|$. Clearly, two cycles $R_{c 1}$ and $R_{c 2}$ carry different signs. Then, let $R_{c 1}^{\prime}=R_{c 1}^{\left|R_{c 2}\right|}$ denote a cycle of node $i$, by continuing $R_{c 1}$ for $\left|R_{c 2}\right|$ times, which has the same sign with $R_{c 1}$ because $\left|R_{c 2}\right|$ is odd. Similarly, we construct a cycle $R_{c 2}^{\prime}=R_{c 2}^{\left|R_{c 1}\right|}$ by continuing $R_{c 2}$ for $\left|R_{c 1}\right|$ times, which has the same sign as $R_{c 2}$. Thus, $R_{c 1}^{\prime}$ and $R_{c 2}^{\prime}$ have the same length of $\left|R_{c 1}\right|\left|R_{c 2}\right|$ but different signs, which contradicts Statement 1 .

2. Proof for

$$
\text { Statement } 2 \Rightarrow \text { Statement } 3 \text {. }
$$

By Proposition A.1 in Appendix A, we know that between any two nodes there must exist even-length paths. By Statement 2, we partition $V$ into $S$ and $\bar{S}$, based on the signs of even-length paths originated from a particular node $i \in V$. More specifically, $S$ contains the nodes to which all even-length paths from $i$ have positive signs, and $\bar{S}$ contains the other set of nodes (note that $i$ may not be in $S$ ). 
We argue that (1) within $S$ and $\bar{S}$, all edges have same signs; and (2) all edges between $S$ and $\bar{S}$ have the same signs. Because $G$ contains both negative and positive edges, it must be either balanced or antibalanced.

For (1), assume to the contrary that there exist two directed edges $R_{a b}=a \rightarrow b$ and $R_{c d}=c \rightarrow d$, which both reside in the same set, e.g., $S$ with different signs. (The case for $\bar{S}$ is similar.)

We construct two paths of even length from $i$ to $c$ and $i$ to $d$ as follows.

$$
\begin{aligned}
& R_{e}(i, c)=R_{e}(i, b)+R_{e}(b, c), \\
& R_{e}(i, d)=R_{e}(i, a)+R_{a b}+R_{e}(b, c)+R_{c d},
\end{aligned}
$$

where $R_{e}(x, y)$ represents the constructed even-length path from node $x$ to node $y$.

Because both $c, d \in S$, by construction, then $R_{e}(i, c)$ and $R_{e}(i, d)$ have the same signs

$$
\operatorname{sgn}\left(R_{e}(i, c)\right)=\operatorname{sgn}\left(R_{e}(i, d)\right) .
$$

Conversely, because $a$ and $b$ are in the same group as $c$ and $d, \operatorname{sgn}\left(R_{e}(i, a)\right)=\operatorname{sgn}\left(R_{e}(i, b)\right)$. Then we have

$$
\begin{aligned}
\operatorname{sgn}\left(R_{e}(i, c)\right) & =\operatorname{sgn}\left(R_{e}(i, b)\right) \operatorname{sgn}\left(R_{e}(b, c)\right), \\
\operatorname{sgn}\left(R_{e}(i, d)\right) & =\operatorname{sgn}\left(R_{e}(i, a)\right) \operatorname{sgn}\left(R_{a b}\right) \operatorname{sgn}\left(R_{e}(b, c)\right) \operatorname{sgn}\left(R_{c d}\right) \\
& =-\operatorname{sgn}\left(R_{e}(i, b)\right) \operatorname{sgn}\left(R_{e}(b, c)\right) .
\end{aligned}
$$

Equation (3.6) comes from the assumption that $R_{a b}$ and $R_{c d}$ have different signs; (3.4) contradicts (3.5) and (3.6).

For (2), assume that there exist two edges $R_{a b}$ and $R_{c d}$ with different signs between $S$ and $\bar{S}$. Still consider the two even-length paths $R_{e}(i, c)$ and $R_{e}(i, d)$ constructed before. Because $c$ and $d$ are not in the same side, $R_{e}(i, c)$ and $R_{e}(i, d)$ have opposite signs by the construction, i.e.,

$$
\operatorname{sgn}\left(R_{e}(i, c)\right)=-\operatorname{sgn}\left(R_{e}(i, d)\right) .
$$

However, because $a$ and $b$ are in the different groups as well, $\operatorname{sgn}\left(R_{e}(i, a)\right)=$ $-\operatorname{sgn}\left(R_{e}(i, b)\right)$. Then we have

$$
\begin{aligned}
\operatorname{sgn}\left(R_{e}(i, c)\right) & =\operatorname{sgn}\left(R_{e}(i, b)\right) \cdot \operatorname{sgn}\left(R_{e}(b, c)\right), \\
\operatorname{sgn}\left(R_{e}(i, d)\right) & =\operatorname{sgn}\left(R_{e}(i, a)\right) \operatorname{sgn}\left(R_{a b}\right) \operatorname{sgn}\left(R_{e}(b, c)\right) \operatorname{sgn}\left(R_{c d}\right) \\
& =\operatorname{sgn}\left(R_{e}(i, b)\right) \cdot \operatorname{sgn}\left(R_{e}(b, c)\right) .
\end{aligned}
$$

However, (3.7) contradicts with (3.8) and (3.9). This completes the proof.

The next lemma characterizes the limiting behavior of $P^{t}$ of ergodic signed digraphs with all three balance structures. Given a signed digraph $G=(V, E, A)$, let $\bar{G}=(V, E, \bar{A})$ corresponds to its unsigned version $\left(\bar{A}_{i j}=\left|A_{i j}\right|\right.$ for all $\left.i, j \in V\right)$. When $\bar{G}$ is ergodic, a random walk on $\bar{G}$ has a unique stationary distribution, denoted as $\pi$. That is, $\pi^{T}=\pi^{T} \bar{P}$, where $\pi^{T}$ is the transpose of the stationary distribution vector $\pi$, and $\bar{P}=D^{-1} \bar{A}$ is the transition probability matrix for $\bar{G}$. Henceforth, we always use $S, \bar{S}$ to denote the 
corresponding partition for either balanced graphs or antibalanced graphs. We define the infinity norm of a matrix $M \in \mathbb{R}^{m \times m}$ as: $\|M\|_{\infty}:=\max _{1 \leq i \leq m} \sum_{j=1}^{m}\left|M_{i j}\right|$.

Lemma 3.6. Given an ergodic signed digraph $G=(V, E, A)$, when $G$ is balanced or strictly unbalanced, $P^{t}$ converges, and when $G$ is antibalanced, the odd and even subsequences of $P^{t}$ converge, respectively.

Balanced $G: \quad \quad \lim _{t \rightarrow \infty} P^{t}=\hat{\mathbf{I}}_{S} \hat{\pi}_{S}^{T} ;$

Strictly unbalanced $G$ : $\quad \lim _{t \rightarrow \infty} P^{t}=\mathbf{0}$;

Antibalanced $G: \quad \lim _{t \rightarrow \infty} P^{2 t}=\hat{\boldsymbol{I}}_{S} \hat{\pi}_{S}^{T}, \lim _{t \rightarrow \infty} P^{2 t+1}=-\hat{\boldsymbol{I}}_{S} \hat{\pi}_{S}^{T}$.

\section{Proof.}

1. When $G$ is balanced, the signed transition matrix $P$ can be written as $P=\hat{I}_{S} \bar{P}_{S}$. Because $\bar{G}$ is ergodic, we have $\lim _{t \rightarrow \infty} \bar{P}^{t}=\mathbf{1} \pi^{T}$. Thus,

$$
\lim _{t \rightarrow \infty} P^{t}=\lim _{t \rightarrow \infty}\left(\hat{I}_{S} \bar{P} \hat{I}_{S}\right)^{t}=\hat{\mathbf{1}}_{S} \hat{\pi}_{S}^{T},
$$

where we use the simple facts $\hat{I}_{S}^{2}=I, \hat{I}_{S} \mathbf{1}=\hat{\mathbf{1}}_{S}$, and $\pi^{T} \hat{I}_{S}=\hat{\pi}_{S}^{T}$.

2. When $G$ is antibalanced, we have $P=-\hat{I}_{S} \bar{P} \hat{I}_{S}$. Thus,

$$
\begin{aligned}
\lim _{t \rightarrow \infty} P^{2 t} & =\lim _{t \rightarrow \infty}\left(-\hat{I}_{S} \bar{P} \hat{I}_{S}\right)^{2 t}=\hat{\mathbf{1}}_{S} \hat{\pi}_{S}^{T} \\
\lim _{t \rightarrow \infty} P^{2 t+1} & =\lim _{t \rightarrow \infty}\left(-\hat{I}_{S} \bar{P} \hat{I}_{S}\right)^{2 t+1}=-\hat{\mathbf{1}}_{S} \hat{\pi}_{S}^{T} .
\end{aligned}
$$

3. When $G$ is strictly unbalanced, by Proposition 3.5, there exists a pair of nodes $i$ and $j$, such that two paths $R_{1}$ and $R_{2}$ from $i$ to $j$ have the same length and opposite signs. Consider node $j$ fixed; there may exist a node other than $i$, having two paths to $j$ with the same length and opposite signs. We denote the length of such paths as $\ell(i)$ with respect to the origin $i \in V$. Given a node $i$, consider a random walk from $i$ to $j$. Let $p_{1}>0$ (resp. $p_{2}>0$ ) be the probability that the walk exactly follows $R_{1}$ (resp. $R_{2}$ ) in the first $\ell(i)$ steps. Let $R_{i, k}^{\ell(i)}$ be the set of all paths from $i$ to $k$ with length $\ell(i)$. Then, for a unit vector $e_{i}$ with $i$ th entry equal to 1 and other entries as 0 , we have $\left\|e_{i}^{T} P^{\ell(i)}\right\|_{1}$ as the sum of signed probabilities by randomly walking from node $i$ to node $j$, which is bounded as follows:

$$
\left\|e_{i}^{T} P^{\ell(i)}\right\|_{1}=\sum_{k \in V}\left|\sum_{R \in R_{i, k}^{\ell(i)}} \operatorname{Prob}[R] \operatorname{sgn}(R)\right| \leq 1-\min \left(p_{1}, p_{2}\right),
$$

where $\operatorname{Prob}[R]$ is the probability that the random walker takes exactly the path $P$, and the inequality holds because of the sign difference between $p_{1}$ and $p_{2}$. We denote the upper bound as $\rho_{i}:=1-\min \left(p_{1}, p_{2}\right)$.

For any node $i^{\prime} \in V$, there must exist a path $R^{\prime}$ from $i^{\prime} \rightarrow i$, because of the ergodicity of $G$ (where $R^{\prime}$ is empty when $i^{\prime}=i$ ), thus, two paths $R_{1}^{\prime}=R^{\prime}+R_{1}$ and $R_{2}^{\prime}=R^{\prime}+R_{2}$ from $i^{\prime}$ to $j$ have the same length, but opposite signs. With similar arguments as that for the node 
$i,\left\|e_{i^{\prime}}^{T} P^{\ell\left(i^{\prime}\right)}\right\|_{1} \leq \rho_{i^{\prime}}$ holds for any $i^{\prime} \in V$. Let $\rho:=\max _{i^{\prime} \in V} \rho_{i^{\prime}}<1$ and $\ell:=\max _{i^{\prime} \in V} \ell\left(i^{\prime}\right)$; we conclude that $\left\|e_{i^{\prime}}^{T} P^{\ell}\right\|_{1} \leq \rho$. We can express the identity matrix $I$ as rows of $e_{i}$ 's, i.e., $I=\left[e_{1}^{T} ; \cdots ; e_{|V|}^{T}\right]$, which infers the following inequality:

$$
\left\|P^{\ell}\right\|_{\infty}=\left\|I P^{\ell}\right\|_{\infty}=\max _{i^{\prime} \in V}\left\|e_{i^{\prime}}^{T} P^{\ell}\right\|_{1} \leq \max _{i^{\prime} \in V} \rho_{i^{\prime}}=\rho<1 .
$$

Hence, by applying the fact that $\|A B\|_{\infty} \leq\|A\|_{\infty}\|B\|_{\infty}$ for any square matrices $A$ and $B$, when $t \geq T=2 \ell$, the following inequality holds

$$
\left\|P^{t}\right\|_{\infty}=\left\|P^{\frac{t}{\ell} \ell}\right\|_{\infty} \leq \rho^{\left\lfloor\frac{t}{\ell}\right\rfloor} \leq \rho^{\frac{t}{T}}
$$

which infers $\lim _{t \rightarrow \infty}\left\|P^{t}\right\|_{\infty}=0$, i.e., $\lim _{t \rightarrow \infty} P^{t}=\mathbf{0}$.

Lemma 3.6 clearly shows different convergence behaviors of $P^{t}$ for three types of graphs. In particular, $P^{t}$ of antibalanced graphs exhibits a bounded oscillating behavior over the long term.

3.2.2. Case of Weakly Connected Signed Digraphs. Now, we consider a weakly connected signed digraph $G=(V, E, A)$ with one ergodic sink component $G_{Z}$ with node set $Z$, which has only incoming edges from the rest of the signed digraph $G_{X}$ with node set $X=V \backslash Z$. Then, the signed transition matrix $P$ has the following block form.

$$
P=\left[\begin{array}{c:c}
P_{X} & P_{Y} \\
\hdashline \mathbf{0} & P_{Z}^{-}
\end{array}\right],
$$

where $P_{X}$ and $P_{Z}$ are the block matrices for component $G_{X}$ and $G_{Z}$, and $P_{Y}$ represents the one-way connections from $G_{X}$ to $G_{Z}$. Then, the $t$-step transition matrix $P^{t}$ can be expressed as

$$
P^{t}=\left[\begin{array}{c:c}
P_{X}^{(t)} & P_{Y}^{(t)} \\
\hdashline \frac{1}{\mathbf{0}} & P_{Z}^{(t)^{-}}
\end{array}\right],
$$

where $P_{X}^{(t)}=P_{X}^{t}, P_{Z}^{(t)}=P_{Z}^{t}$ and $P_{Y}^{(t)}=\sum_{i=0}^{t-1} P_{X}^{i} P_{Y} P_{Z}^{t-1-i}$. When $G_{Z}$ is balanced or antibalanced, we use $S_{Z}, \bar{S}_{Z}$ to denote the partition of $Z$ defining its balance or antibalance structure. Then, we denote column vectors

$$
\begin{aligned}
u_{b} & =\left(I_{X}-P_{X}\right)^{-1} P_{Y} \hat{\mathbf{1}}_{Z, S_{Z}}, \\
\text { and } u_{u} & =\left(I_{X}+P_{X}\right)^{-1} P_{Y} \hat{\mathbf{1}}_{Z, S_{Z}} .
\end{aligned}
$$

The reason that $I_{X}-P_{X}$ is invertible is because $\lim _{t \rightarrow \infty} P_{X}^{t}=\mathbf{0}$, which is in turn because there is a path from any node $i$ in $G_{X}$ to nodes in $Z$ (because $Z$ is the single sink), and, thus, informally a random walk from $i$ eventually reaches and then stays in $G_{Z}$. The same reason applies to $I_{X}+P_{X}$. Lemma 3.7 provides the formal proof of the fact $\lim _{t \rightarrow \infty} P_{X}^{t}=\mathbf{0}$.

Let $\pi_{Z}$ denote the stationary distribution of nodes in $G_{Z}$, and $\hat{\pi}_{Z, S_{Z}}$ is signed, with $\hat{\pi}_{Z, S_{Z}}(i)=\pi_{Z}(i)$ for $i \in S_{Z}$, and $\hat{\pi}_{Z, S_{Z}}(i)=-\pi_{Z}(i)$ for $i \in Z \backslash S_{Z}$. Lemma 3.7 describes the convergence of $P^{t}$ given various balance structures of $G_{Z}$. 
Lemma 3.7. For weakly connected signed digraph $G=(V, E, A)$ with one ergodic sink component, with signed transition matrix given in (3.11), we have

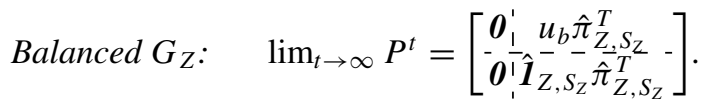

$$
\begin{aligned}
& \text { Strictly unbalanced } G_{Z} \text { : } \quad \lim _{t \rightarrow \infty} P^{t}=\mathbf{0} \text {. } \\
& \text { Antibalanced } G_{Z}: \quad \lim _{t \rightarrow \infty} P^{2 t}=\left[\begin{array}{c}
\boldsymbol{O}_{1}^{1}-u_{u} \hat{\pi}_{Z, S_{Z}}^{T} \\
\hdashline \mathbf{0}_{1}^{\prime} \hat{\boldsymbol{1}}_{Z, S_{Z}}^{-} \hat{\pi}_{Z, S_{Z}}^{T}
\end{array}\right], \\
& \lim _{t \rightarrow \infty} P^{2 t+1}=\left[\begin{array}{cc}
\boldsymbol{0}_{1}^{1} & u_{u} \hat{\pi}_{Z, S_{Z}}^{T} \\
\hdashline \boldsymbol{0} & -\hat{\boldsymbol{1}}_{Z, S_{Z}}^{-} \hat{\pi}_{Z, S_{Z}}^{T}
\end{array}\right] .
\end{aligned}
$$

Proof. We discuss the convergence of $P_{X}^{t}, P_{Z}^{t}$, and $P_{Y}^{(t)}$ in (3.11).

1. We first prove that $P_{X}^{t}$ converges to $\mathbf{0}$, i.e., $\lim _{t \rightarrow \infty} P_{X}^{t}=\mathbf{0}$.

Since $G_{X}$ does not contain sink components, any node $i \in X$ has a path to component $G_{Z}$. Let $R_{i Z}$ be the shortest path from $i$ to some node in $Z$, and $\operatorname{Prob}\left[R_{i Z}\right]$ denote the probability that a random walk starting from $i$ takes the path $R_{i Z}$. Hence, we denote

$$
p=\min _{i \in X} \operatorname{Prob}\left[R_{i Z}\right], \text { and } m=\max _{i \in X}\left|R_{i Z}\right|,
$$

which implies that starting from any node $i \in X$, after $m$ steps of random walk, there is at least probability $p$ that it reaches component $G_{Z}$. Hence, we have $\left\|P_{X}^{m}\right\|_{\infty} \leq(1-p)<1$. Let $T=2 m$, then for any $t>T$, we have

$$
\left\|P_{X}^{t}\right\|_{\infty}=\left\|P_{X}^{\frac{t}{m} m}\right\|_{\infty} \leq(1-p)^{\left\lfloor\frac{t}{m}\right\rfloor} \leq(1-p)^{\frac{t}{T}},
$$

which implies $\lim _{t \rightarrow \infty}\left\|P_{X}^{t}\right\|_{\infty}=0$, i.e., $\lim _{t \rightarrow \infty} P_{X}^{t}=\mathbf{0}$.

2. For subgraph $G_{Z}$, Lemma 3.6 directly yields

$$
\lim _{t \rightarrow \infty} P_{Z}^{t}= \begin{cases}\mathbf{0}, & \text { Strictly unbalanced } G_{Z} \\ \mathbf{1}_{Z, S_{Z}} \pi_{Z, S_{Z}}^{T}, & \text { Balanced } G_{Z} ; \\ \mathbf{1}_{Z, S_{Z}} \pi_{Z, S_{\mathcal{Z}}}^{T}, & \text { Antibalanced } G_{Z}, \text { even } t \\ -\mathbf{1}_{Z, S_{Z}} \pi_{Z, S_{Z}}^{T}, & \text { Antibalanced } G_{Z}, \text { odd } t\end{cases}
$$

3. Below, we focus on proving the results on $\lim _{t \rightarrow \infty} P_{Y}^{(t)}$ using Proposition B.1 in Appendix B.

When $G_{Z}$ is strictly unbalanced, from Lemma 3.6 and (3.1) in this proof, $\lim _{t \rightarrow \infty} P_{X}^{t}=\mathbf{0}$ and $\lim _{t \rightarrow \infty} P_{Z}^{t}=\mathbf{0}$ hold, thus, by Proposition B.1 in Appendix B $\lim _{t \rightarrow \infty} P_{Y}^{(t)}=\mathbf{0}$.

When $G_{Z}$ is balanced, Lemma 3.6 and Proposition A.2 in Appendix A directly yield $\left(P_{Z}-\mathbf{1}_{Z, S_{Z}} \pi_{Z, S_{Z}}^{T}\right)^{t}=P_{Z}^{t}-\mathbf{1}_{Z, S_{Z}} \pi_{Z, S_{Z}}^{T}$ for any integer $t>0$, and $\lim _{t \rightarrow \infty}$ 
$\left(P_{Z}-\mathbf{1}_{Z, S_{Z}} \pi_{Z, S_{Z}}^{T}\right)^{t}=\mathbf{0}$, thus,

$$
\begin{aligned}
& \lim _{t \rightarrow \infty} P_{Y}^{(t)}=\lim _{t \rightarrow \infty} \sum_{i=0}^{t-1} P_{X}^{i} P_{Y}\left(P_{Z}^{t-1-i}-\mathbf{1}_{Z, S_{Z}} \pi_{Z, S_{Z}}^{T}+\mathbf{1}_{Z, S_{Z}} \pi_{Z, S_{Z}}^{T}\right) \\
& =\lim _{t \rightarrow \infty} \sum_{i=0}^{t-1} P_{X}^{i} P_{Y}\left(P_{Z}-\mathbf{1}_{Z, S_{Z}} \pi_{Z, S_{Z}}^{T}\right)^{t-1-i}+\lim _{t \rightarrow \infty} \sum_{i=0}^{t-2} P_{X}^{i} P_{Y} \mathbf{1}_{Z, S_{Z}} \pi_{Z, S_{Z}}^{T} \\
& =\left(I_{X}-P_{X}\right)^{-1} P_{Y} \mathbf{1}_{Z, S_{Z}} \pi_{Z, S_{Z}}^{T}=u_{b} \pi_{Z, S_{Z}}^{T},
\end{aligned}
$$

where the first term in the second line being $\mathbf{0}$ is due to Proposition B.1 (ii) in Appendix B.

When $G_{Z}$ is antibalanced, applying Lemma 3.6 and Proposition A.2 in Appendix A, we have for any integer $t>0,\left(P_{Z}+\mathbf{1}_{Z, S_{Z}} \pi_{Z, S_{Z}}^{T}\right)^{t}=P_{Z}^{t}-(-1)^{t} \mathbf{1}_{Z, S_{Z}} \pi_{Z, S_{Z}}^{T}$, and $\lim _{t \rightarrow \infty}\left(P_{Z}+\right.$ $\left.\mathbf{1}_{Z, S_{Z}} \pi_{Z, S_{Z}}^{T}\right)^{t}=\mathbf{0}$ hold true, thus,

$$
\begin{aligned}
& \lim _{t \rightarrow \infty} P_{Y}^{(t)}=\lim _{t \rightarrow \infty} \sum_{i=0}^{t-1} P_{X}^{i} P_{Y}\left(P_{Z}^{t-1-i}-(-1)^{t-1-i}\left(\mathbf{1}_{Z, S_{Z}} \pi_{Z, S_{Z}}^{T}-\mathbf{1}_{Z, S_{Z}} \pi_{Z, S_{Z}}^{T}\right)\right) \\
& =\lim _{t \rightarrow \infty} \sum_{i=0}^{t-1} P_{X}^{i} P_{Y}\left(P_{Z}+\mathbf{1}_{Z, S_{Z}} \pi_{Z, S_{Z}}^{T}\right)^{t-1-i}+\lim _{t \rightarrow \infty} \sum_{i=0}^{t-2}(-1)^{t-1-i} P_{X}^{i} P_{Y} \mathbf{1}_{Z, S_{Z}} \pi_{Z, S_{Z}}^{T} \\
& =(-1)^{t-1} \lim _{t \rightarrow \infty} \sum_{i=0}^{t-2}\left(-P_{X}\right)^{i} P_{Y} \mathbf{1}_{Z, S_{Z}} \pi_{Z, S_{Z}}^{T}=(-1)^{t-1}\left(I_{X}+P_{X}\right)^{-1} P_{Y} \mathbf{1}_{Z, S_{Z}} \pi_{Z, S_{Z}}^{T} \\
& =(-1)^{t-1} u_{u} \pi_{Z, S_{Z}}^{T} .
\end{aligned}
$$

Hence, we have for antibalanced $G_{Z}: \lim _{t \rightarrow \infty} P_{Y}^{(2 t)}=-u_{u} \hat{\pi}_{Z, S_{Z}}^{T}$, and $\lim _{t \rightarrow \infty} P_{Y}^{(2 t+1)}=$ $u_{u} \hat{\pi}_{Z, S_{Z}}^{T}$.

\subsubsection{Multiple Sink Components and Disconnected Signed Digraphs.}

When there exist $m>1$ ergodic sink components, i.e., $G_{Z 1}, G_{Z 2}, \cdots, G_{Z m}$, the rest of the graph $G$ is considered as $G_{X}$. Then the signed transition matrix $P$ and $P^{t}$ can be written as

$$
P=\left[\begin{array}{c:c:c:c}
P_{X} & P_{Y 1} & \ldots & P_{Y m} \\
\hdashline \mathbf{0} & P_{Z 1} & \mathbf{0} & \mathbf{0} \\
\hdashline \mathbf{0} & \mathbf{0} & \ddots & \mathbf{0} \\
\hdashline \mathbf{0} & \mathbf{0} & \mathbf{0} & P_{Z m}
\end{array}\right], \quad P^{t}=\left[\begin{array}{c:c:c:c}
P_{X}^{t} & P_{Y}^{(t)} & \ldots & P_{Y m}^{(t)} \\
\hdashline \mathbf{0} & P_{Z}^{t} & \mathbf{0} & \mathbf{0} \\
\hdashline \mathbf{0} & \mathbf{0} & \ddots & \mathbf{0} \\
\hdashline \mathbf{0} & \mathbf{0} & \mathbf{0} & P_{Z m}^{t}
\end{array}\right],
$$

where $P_{Y i}^{(t)}=\sum_{j=0}^{t-1} P_{X}^{j} P_{Y i} P_{Z i}^{t-1-j}, 1 \leq i \leq m$. Hence, each sink ergodic component $P_{Z i}$ along with $P_{X}$ and $P_{Y i}$ independently follows Lemma 3.7. For disconnected signed digraph, with $m \geq 1$ ergodic or weakly connected components, each of which satisfies Lemma 3.6 or Lemma 3.7, respectively. For brevity, we omit the details here. 


\subsection{Long-Term Dynamics}

Based on the structural balance classification and the convergence of signed transition matrix discussed above, we are ready now to analyze the long-term dynamics of the voter model on signed digraphs. Formally, we are interested in characterizing $x_{t}$ with $t \rightarrow \infty$, i.e.,

$$
x=\lim _{t \rightarrow \infty} x_{t}=\lim _{t \rightarrow \infty}\left(P^{t} x_{0}+\left(\sum_{i=0}^{t-1} P^{i}\right) g^{-}\right) .
$$

If the even and odd subsequences of $x_{t}$ converge separately, we denote $x_{e}=$ $\lim _{t \rightarrow \infty} x_{2 t}, x_{o}=\lim _{t \rightarrow \infty} x_{2 t+1}$.

Before presenting the results on the long-term dynamics of the voter model, we first introduce the following useful lemma connecting a signed digraph $G$ with another graph $G^{\prime}$ where all edge signs in $G$ are negated.

Lemma 3.8. Given a signed digraph $G=(V, E, A)$, let $G^{\prime}=(V, E,-A)$ be a signed digraph with all edge signs negated from $G$. Then, for any initial color distribution $x_{0}$, at any $2 t$ steps $(t>0)$, the color distributions $x_{2 t}(G)$ on $G$ and $x_{2 t}\left(G^{\prime}\right)$ on $G^{\prime}$ are identical.

Proof. Let $P^{\prime}=-P$ denote the signed transition matrix of $G^{\prime}$, and denote the vector $g^{-}=D^{-1} A^{-1}$ and $g^{\prime-}=D^{-1}(-A)^{-} \mathbf{1}=D^{-1} A^{+} \mathbf{1}$. Thus $g^{\prime-}=\mathbf{1}-g^{-}$. By (3.1), after two steps, we have

$$
\begin{aligned}
x_{2}\left(G^{\prime}\right)=P^{\prime 2} x_{0}+P^{\prime} g^{\prime-}+g^{\prime-} & =P^{2} x_{0}-P\left(\mathbf{1}-g^{-}\right)+\mathbf{1}-g^{-} \\
& =P^{2} x_{0}+P g^{-}+g^{-}=x_{2}(G),
\end{aligned}
$$

where the last equality uses facts $\mathbf{1}=D^{-1} \bar{A} \mathbf{1}$ and $P=D^{-1} A$. Since the lemma holds for two steps, then clearly it holds for all even steps.

The next theorem discusses the case of ergodic signed digraphs.

Theorem 3.9. Let $G=(V, E, A)$ be an ergodic signed digraph, we have

$$
\begin{array}{rc}
\text { Balanced } G: & x=\hat{\boldsymbol{I}}_{S} \hat{\pi}_{S}^{T}\left(x_{0}-\frac{1}{2} \boldsymbol{l}\right)+\frac{1}{2} \boldsymbol{l} \\
\text { Strictly unbalanced } G: & x=\frac{1}{2} \boldsymbol{l} \\
\text { Anti-balanced } G: & x_{e}=\hat{\boldsymbol{I}}_{S} \hat{\pi}_{S}^{T}\left(x_{0}-\frac{1}{2} \boldsymbol{l}\right)+\frac{1}{2} \boldsymbol{l} \\
& x_{o}=-\hat{\boldsymbol{I}}_{S} \hat{\pi}_{S}^{T}\left(x_{0}-\frac{1}{2} \boldsymbol{l}\right)+\frac{1}{2} \boldsymbol{l}
\end{array}
$$

Proof. We discuss the limit in Eq. (3.16) for three possible balance structures of $G$.

Balanced digraphs. From Lemma 3.6 and Proposition A.2 in Appendix A, it is easy to prove $P^{m}-\hat{\mathbf{1}}_{S} \hat{\pi}_{S}^{T}=\left(P-\hat{\mathbf{1}}_{S} \hat{\pi}_{S}^{T}\right)^{m}$ for any integer $m>0$, which yields the following result 
on the second part in (3.16).

$$
\begin{aligned}
\lim _{t \rightarrow \infty} \sum_{i=0}^{t-1} P^{i} g^{-} & =\left(I-P+\hat{\mathbf{1}}_{S} \hat{\pi}_{S}^{T}\right)^{-1} g^{-}+\lim _{t \rightarrow \infty} \sum_{i=1}^{t-1} \mathbf{1}_{S} \hat{\pi}_{S}^{T} g^{-} \\
& =\left(I-P+\hat{\mathbf{1}}_{S} \hat{\pi}_{S}^{T}\right)^{-1} g^{-}=\frac{1}{2} \mathbf{1}-\frac{1}{2} \hat{\mathbf{1}}_{S} \hat{\pi}_{S}^{T} \mathbf{1},
\end{aligned}
$$

where the last term of (3.21) is canceled because of the digraph flow circulation law [12,30], i.e.,

$$
\hat{\pi}_{S}^{T} g^{-}=\hat{\pi}_{S}^{T} D^{-1} A^{-} \mathbf{1}=\sum_{i \in S} \pi(i) \sum_{j \in \bar{S}} \bar{P}_{i j}-\sum_{i \in \bar{S}} \pi(i) \sum_{j \in S} \bar{P}_{i j}=0 .
$$

The last equality in (3.22) holds because

$$
\frac{1}{2}\left(I-P+\hat{\mathbf{1}}_{S} \hat{\pi}_{S}^{T}\right)\left(\mathbf{1}-\hat{\mathbf{1}}_{S} \hat{\pi}_{S}^{T} \mathbf{1}\right)-g^{-}=0 .
$$

Equation (3.17) is obtained by combining (3.22) with Lemma 3.6.

Antibalanced Digraphs. Lemma 3.8 directly yields (3.19). The odd step influence distribution sequence is obtained by

$$
x_{o}=P x_{e}+g^{-}=-\mathbf{1}_{S} \hat{\pi}_{S}^{T}\left(x_{0}-\frac{1}{2} \mathbf{1}\right)+\frac{1}{2} \mathbf{1} .
$$

Strictly unbalanced digraphs. From Theorem $3.9, \lim _{t \rightarrow \infty} P^{t}=\mathbf{0}$ holds, and thus we have

$$
\lim _{t \rightarrow \infty} \sum_{i=0}^{t-1} P^{i} g^{-}=(I-P)^{-1} g^{-}=(D-A)^{-1} A^{-} \mathbf{1}=\frac{1}{2} \mathbf{1} .
$$

The last equality comes from the fact that $(D-A) \mathbf{1}=2 A^{-} \mathbf{1}$.

Theorem 3.9 has several implications. First, for strictly unbalanced digraphs, each node has equal steady state probability of being black or white, and it is not determined by the initial distribution $x_{0}$. Second, antibalanced digraphs have the same steady state distribution as the corresponding balanced graph for even steps, and for odd steps, the distribution oscillates to the opposite $\left(x_{o}=\mathbf{1}-x_{e}\right)$. Moreover, (3.17) can also be intuitively explained from the random walk interpretation of the voter model. In particular, starting from node $i$, if we perform a random walk for an infinite number of steps, the probability that the random walk stops at $j$ is given by the stationary distribution $\pi(j)$. For balanced graphs, if $i$ and $j$ are from the same component (either $S$ or $\bar{S}$ ), then the random walk must pass an even number of negative edges, so $i$ takes the same color as $j$; if $i$ and $j$ are from opposite components, then the walk passes an odd number of negative edges and $i$ takes the opposite of $j$ 's color. Thus, the steady distribution of $i \in S$ being white is given by $\pi_{S}^{T} x_{0 S}+\pi_{\bar{S}}^{T}\left(\mathbf{1}_{\bar{S}}-x_{0 \bar{S}}\right)$, and the case of $i \in \bar{S}$ is symmetric. Some algebra manipulations can lead us to (3.17).

For a balanced ergodic digraph $G$ with partition $S, \bar{S}$, it is easy to check that it has the following two equilibrium states: in one state all nodes in $S$ are white, and all nodes in $\bar{S}$ are black; and in the other state all nodes in $S$ are black and all nodes in $\bar{S}$ are white. We 
call these two states the polarized states. Using random walk interpretation, we show in the following theorem that with probability 1 , the voter model dynamic converges to one of the previous two equilibrium states.

Theorem 3.10. Given an ergodic signed digraph $G=(V, E, A)$, if $G$ is balanced with partition $S, \bar{S}$, the voter model dynamic converges to one of the polarized states with probability 1, and the probability of nodes in $S$ being white is $\hat{\pi}_{S}^{T}\left(x_{0}-\frac{1}{2} \boldsymbol{I}\right)+\frac{1}{2}$. Similarly, if $G$ is antibalanced, with probability 1 the voter model dynamic oscillates between the two polarized states eventually, and the probability of nodes in $S$ being white at even steps is $\hat{\pi}_{S}^{T}\left(x_{0}-\frac{1}{2} \boldsymbol{l}\right)+\frac{1}{2}$.

Proof. Consider a balanced ergodic digraph $G$ with partition $S, \bar{S}$. By ergodicity, given any two nodes $i$ and $j$, with probability 1 the random walks starting from $i$ and $j$ will meet eventually. If $i$ and $j$ are both in $S$, when the two walks meet at some node $u$, they both pass either an even number of negative edges (if $u \in S$ ) or an odd number of negative edges (if $u \in \bar{S}$ ). Therefore, $i$ and $j$ must be in the same color with probability 1 . If $i$ and $j$ are from different components $S$ and $\bar{S}$, a similar argument shows that they will have the opposite color with probability 1 . Therefore the final state is one of the two polarized states. The probability of nodes in $S$ being white is simply given by Theorem 3.9, (3.17). The case of antibalanced ergodic digraphs can be argued in a similar way.

Theorem 3.11 introduces the long-term dynamics of the weakly connected signed digraphs. We consider weakly connected $G$ with a single sink ergodic component $G_{Z}$ and use the same notations as in Section 3.2.

Theorem 3.11. Let $G=(V, E, A)$ be a weakly connected signed digraph with a single sink component $G_{Z}$ and a nonsink component $G_{X}$. The long-term white color distribution vector $x$ is expressed in two parts:

$$
x^{T}=\lim _{t \rightarrow \infty} x_{t}^{T}=\left[x_{X Y}^{T}, x_{Z}^{T}\right],
$$

where $x_{Z}$ is the limit of $x_{t Z}$ on $G_{Z}$ with initial distribution $x_{0 Z}$ and is given as in Theorem 3.9, and vector $x_{X Y}$ is given below with respect to the balance structure of $G_{Z}$ :

$$
\begin{array}{rc}
\text { Balanced } G_{Z}: & x_{X Y}=\frac{1}{2} \boldsymbol{1}_{X}+u_{b} \hat{\pi}_{Z, S_{Z}}^{T}\left(x_{0 Z}-\frac{1}{2} \boldsymbol{1}_{Z}\right), \\
\text { Strictly unbalanced } G_{Z}: & x_{X Y}=\frac{1}{2} \boldsymbol{1}_{X}, \\
\text { Antibalanced } G_{Z}, \text { even } t: & x_{X Y, e}=\frac{1}{2} \boldsymbol{1}_{X}-u_{u} \hat{\pi}_{Z, S_{Z}}^{T}\left(x_{0 Z}-\frac{1}{2} \boldsymbol{1}_{Z}\right), \\
\text { Antibalanced } G_{Z}, \text { odd } t: & x_{X Y, o}=\frac{1}{2} \boldsymbol{1}_{X}+u_{u} \hat{\pi}_{Z, S_{Z}}^{T}\left(x_{0 Z}-\frac{1}{2} \boldsymbol{1}_{Z}\right),
\end{array}
$$

where $u_{b}$ and $u_{u}$ are defined in (3.12) and (3.13).

Proof. Let initial distribution $x_{0}^{T}=\left[x_{0 X}^{T}, x_{0 Z}^{T}\right]$ and $g^{-T}=\left[g_{X}^{-T}, g_{Z}^{-T}\right]$. When $t \rightarrow \infty$, (3.1) can be written as

$$
x^{T}=\lim _{t \rightarrow \infty}\left(P^{t} x_{0}\right)^{T}=\left[x_{X Y}^{T}, x_{Z}^{T}\right]=\left[x_{X}^{T}+x_{Y}^{T}, x_{Z}^{T}\right]
$$


where $x_{X}=\lim _{t \rightarrow \infty}\left(P_{X}^{t} x_{0 X}+\sum_{i=0}^{t-1} P_{X}^{i} g_{X}^{-}\right), x_{Y}=\lim _{t \rightarrow \infty}\left(P_{Y}^{(t)} x_{0 Z}+\sum_{i=0}^{t-1} P_{Y}^{(i)} g_{Z}^{-}\right)$, and $x_{Z}=\lim _{t \rightarrow \infty}\left(P_{Z}^{t} x_{0 Z}+\sum_{i=0}^{t-1} P_{Z}^{i} g_{Z}^{-}\right)$.

From Lemma 3.7, $\lim _{t \rightarrow \infty} P_{X}^{t}=\mathbf{0}$, thus $x_{X}=\left(I_{X}-P_{X}\right)^{-1} g_{X}^{-}$holds for any ergodic $G_{Z}$. Since $G_{Z}$ is ergodic, $x_{Z}$ follows Theorem 3.9. Following, we will focus on deriving $x_{Y}$, where the first part of $x_{Y}$ satisfies Lemma 3.7, i.e.,

$$
\lim _{t \rightarrow \infty} P_{Y}^{(t)} x_{0 Z}=\left\{\begin{array}{cc}
\mathbf{0} & G_{Z} \text { is strictly unbalanced } \\
u_{b} \hat{\pi}_{Z, S_{Z}}^{T} x_{0 Z} & G_{Z} \text { is balanced } \\
-u_{u} \hat{\pi}_{Z, S_{Z}}^{T} x_{0 Z} & G_{Z} \text { is anti-balanced, even } t \\
u_{u} \hat{\pi}_{Z, S_{Z}}^{T} x_{0 Z} & G_{Z} \text { is anti-balanced, odd } t .
\end{array}\right.
$$

The second part of $x_{Y}$ can be further written as

$$
\begin{aligned}
\lim _{m \rightarrow \infty} \sum_{t=1}^{m} P_{Y}^{(t)} g_{Z}^{-} & =\lim _{m \rightarrow \infty} \sum_{t=0}^{m-1} \sum_{i=0}^{t}\left(P_{X}^{t-i} P_{Y} P_{Z}^{i}\right) g_{Z}^{-} \\
& =\lim _{m \rightarrow \infty} \sum_{t=0}^{m-1} \sum_{i=0}^{m-t}\left(P_{X}^{t} P_{Y} P_{Z}^{i}\right) g_{Z}^{-}=\sum_{t=0}^{\infty}\left(P_{X}^{t} P_{Y} \sum_{i=0}^{\infty} P_{Z}^{i}\right) g_{Z}^{-}
\end{aligned}
$$

Now we discuss (3.24) under different balance structures of $G_{Z}$.

1. $G_{Z}$ is strictly unbalanced. From Lemma $3.7, \lim _{t \rightarrow \infty} P^{t}=\mathbf{0}$. Then by (3.23) we directly obtain that $x_{X Y}=\frac{1}{2} \mathbf{1}_{X}$. Applying (3.23) to $\sum_{i=0}^{\infty} P_{Z}^{i} g_{Z}^{-}$in (3.24), we have

$$
\lim _{m \rightarrow \infty} \sum_{t=1}^{m} P_{Y}^{(t)} g_{Z}^{-}=\frac{1}{2}\left(I_{X}-P_{X}\right)^{-1} P_{Y} \mathbf{1}_{Z}
$$

Thus, we obtain the following equation:

$$
x_{X Y}=x_{X}+x_{Y}=\left(I_{X}-P_{X}\right)^{-1}\left(g_{X}^{-}+\frac{1}{2} P_{Y} \mathbf{1}_{Z}\right)=\frac{1}{2} \mathbf{1}_{X} .
$$

2. $G_{Z}$ is balanced. Using (3.22), we have

$$
\lim _{m \rightarrow \infty} \sum_{t=1}^{m} P_{Y}^{(t)} g_{Z}^{-}=\frac{1}{2}\left(I_{X}-P_{X}\right)^{-1} P_{Y}\left(\mathbf{1}_{Z}-\hat{\mathbf{1}}_{Z, S_{Z}} \hat{\pi}_{Z, S_{Z}}^{T} \mathbf{1}_{Z}\right) .
$$

Hence, we have

$$
\begin{aligned}
x_{X Y} & =\left(I_{X}-P_{X}\right)^{-1}\left(g_{X}^{-}+\frac{1}{2} P_{Y} \mathbf{1}_{Z}\right)+u_{b} \hat{\pi}_{Z, S_{Z}}^{T}\left(x_{0 Z}-\frac{1}{2} \mathbf{1}_{Z}\right) \\
& =\frac{1}{2} \mathbf{1}_{X}+u_{b} \hat{\pi}_{Z, S_{Z}}^{T}\left(x_{0 Z}-\frac{1}{2} \mathbf{1}_{Z}\right) .
\end{aligned}
$$

3. $G_{Z}$ is antibalanced. Using Lemma 3.8 , we can negate the signs of all edges in $G$ so that the sink becomes balanced. Hence, we know that at even steps in long term,

$$
x_{X Y, e}=\frac{1}{2} \mathbf{1}_{X}-u_{u} \hat{\pi}_{Z, S_{Z}}^{T}\left(x_{0 Z}-\frac{1}{2} \mathbf{1}_{Z}\right),
$$


where (3.26) and (3.25) are identical in the sense that $P_{X}$ 's and $P_{Y}$ 's in (3.26) and (3.25) have opposite signs. Moreover, the odd-step influence distribution sequence is obtained

$$
x_{X Y, o}=P_{X} x_{X Y, e}+P_{Y} x_{Z, e}+g_{X}^{-}=\frac{1}{2} \mathbf{1}_{X}+u_{u} \hat{\pi}_{Z, S_{Z}}^{T}\left(x_{0 Z}-\frac{1}{2} \mathbf{1}_{Z}\right) .
$$

Theorem 3.11 characterizes the long-term dynamics when the underlying graph is a weakly connected signed digraph with one ergodic sink component. We can see that the results for balanced and antibalanced sink components are more complicated than the ergodic digraph case, because how nonsink components are connected to the sink subtly affects the final outcome of the steady state behavior. In steady state, while the sink component is still in one of the two polarized states as stated in Theorem 3.10, the nonsink components exhibit more complicated color distribution, for which we provide probability characterizations in Theorem 3.11. Using (3.15), Theorem 3.9 and Theorem 3.11 can be readily extended to the case with more than one ergodic sink components and disconnected digraphs. When the network contains only positive directed edges, the voter model dynamics can be interpreted using digraph random walk theory [28-32].

\section{INFLUENCE MAXIMIZATION}

With the detailed analysis on voter model dynamics for signed digraphs, we are ready now to solve the influence maximization problem. Intuitively, we want to address the following question: If only at most $k$ nodes could be selected initially and be turned white while all other nodes are black, how should we choose seed nodes in order to maximize the expected number of white nodes in short term and in long term, respectively?

\subsection{Influence Maximization Problem}

4.1.1. Influence Maximization Objectives. We consider two types of shortterm influence objectives, one is the instant influence, which counts the total number of influenced nodes at a step $t>0$; the other is the average influence, which takes the average number of influenced nodes within the first $t$ steps. These two objectives have different implications and applications. For example, political campaigns try to convince voters who may change their minds back and forth, but only the voters' opinions on the voting day are counted, which matches the instant influence. However, a credit card company would like to have customers keep using its credit card service as much as possible, which is better interpreted by the average influence. When $t$ is sufficiently large, it becomes the long-term objective, and long-term average influence coincides with long-term instant influence when the dynamic converges.

Formally, we define the short-term instant influence $f_{t}\left(x_{0}\right)$ and the short-term average influence $\bar{f}_{t}\left(x_{0}\right)$ as follows:

$$
f_{t}\left(x_{0}\right):=\mathbf{1}^{T} x_{t}\left(x_{0}\right) \text { and } \bar{f}_{t}\left(x_{0}\right):=\frac{\sum_{i=0}^{t} f_{i}\left(x_{0}\right)}{t+1}
$$


Moreover, we define long-term influence as

$$
f\left(x_{0}\right):=\lim _{t \rightarrow \infty} \frac{\sum_{i=0}^{t} f_{i}\left(x_{0}\right)}{t+1} .
$$

Note that when the dynamic converges (e.g., ergodic balanced or ergodic strictly unbalanced graphs), $f\left(x_{0}\right)=\lim _{t \rightarrow \infty} f_{t}\left(x_{0}\right)$. For ergodic antibalanced graphs (or sink components), it is essentially the average of even- and odd-step limit influence.

Given a set $W \subseteq V$, let $e_{W}$ be the vector in which $e_{W}(j)=1$ if $j \in W$, and $e_{W}(j)=0$ if $j \notin W$, which represents the initial seed distribution with only nodes in $W$ as white seeds. Let $e_{i}$ be the shorthand of $e_{\{i\}}$. Unlike unsigned graphs, if initially no white seeds are selected on a signed digraph $G$, i.e., $x_{0}=\mathbf{0}$, the instant influence $f_{t}(\mathbf{0})$ at step $t$ is in general nonzero, which is referred to as the ground influence of the graph $G$ at $t$. The influence contribution of a seed set $W$ does not count such ground influence, as shown in Definition 4.1.

Definition 4.1. (Influence contribution) The instant influence contribution of a seed set $W$ to the $t$ th step instant influence objective, denoted by $c_{t}(W)$, is the difference between the instant influence at step $t$ with only nodes in $W$ selected as seeds and the ground influence at step $t: c_{t}(W)=f_{t}\left(e_{W}\right)-f_{t}(\mathbf{0})$. The average influence contribution $\bar{c}_{t}(W)$ and long-term influence contribution $c(W)$ are defined in the same way: $\bar{c}_{t}(W)=\bar{f}_{t}\left(e_{W}\right)-\bar{f}_{t}(\mathbf{0})$ and $c(W)=f\left(e_{W}\right)-f(\mathbf{0})$.

We are now ready to formally define the influence maximization problem.

Definition 4.2. (Influence maximization) The influence maximization problem for shortterm instant influence is finding a seed set $W$ of at most $k$ seeds that maximizes $W$ 's instance influence contribution at step $t$, i.e., finding $W_{t}^{*}=\arg \max _{|W| \leq k} c_{t}(W)$. Similarly, the problem for average influence and long-term influence is finding $\bar{W}_{t}^{*}=\arg \max _{|W| \leq k} \bar{c}_{t}(W)$ and $W^{*}=\arg \max _{|W| \leq k} c(W)$, respectively.

We now provide some properties of influence contribution, which lead to the optimal seed selection rule. By (3.1), we have

$$
c_{t}(W)=f_{t}\left(e_{W}\right)-f_{t}(\mathbf{0})=\mathbf{1}^{T} x_{t}\left(e_{W}\right)-\mathbf{1}^{T} x_{t}(\mathbf{0})=\mathbf{1}^{T} P^{t} e_{W} .
$$

Let $c_{t}(i)$ be the shorthand of $c_{t}(\{i\})$, and let $c_{t}=\left[c_{t}(i)\right]$ denote the vector of influence contribution of individual nodes. Then $c_{t}^{T}=\left[c_{t}(i)\right]^{T}=\mathbf{1}^{T} P^{t}$. When $t \rightarrow \infty$, the longterm influence contributions of individual nodes are obtained as a vector $c$ :

$$
c^{T}=\lim _{t \rightarrow \infty} \frac{\sum_{i=0}^{t} c_{i}^{T}}{t+1}=\lim _{t \rightarrow \infty} \frac{\mathbf{1}^{T} \sum_{i=0}^{t} P^{i}}{t+1} .
$$

When $P^{t}$ converges, we simply have

$$
c^{T}=\mathbf{1}^{T} \lim _{t \rightarrow \infty} P^{t} .
$$

Lemma 4.3 below discloses the important property that the influence contribution is a linear set function. 
Lemma 4.3. Given a white seed set $W, c_{t}(W)=\sum_{i \in W} c_{t}(i), \bar{c}_{t}(W)=\sum_{i \in W} \bar{c}_{t}(i)$, and $c(W)=\sum_{i \in W} c(i)$.

Proof. From (4.3), we have

$$
c_{t}(W)=\mathbf{1}^{T} P^{t} e_{W}=\mathbf{1}^{T} P^{t} \sum_{i \in W} e_{i}=\sum_{i \in W} \mathbf{1}^{T} P^{t} e_{i}=\sum_{i \in W} c_{t}(i) .
$$

The linearity of $\bar{c}_{t}$ and $c$ can be derived from that of $c_{t}$.

Given a vector $v$, let $n^{+}(v)$ denote the number of positive entries in $v$. By applying Lemma 4.3, we have the optimal seed selection rule for instant influence maximization as follows.

4.1.2. Optimal Seed Selection Rule for Instant Influence Maximization. Given a signed digraph and a limited budget $k$, selecting top $\min \left\{k, n^{+}\left(c_{t}\right)\right\}$ seeds with the highest $c_{t}(i)$ 's, $i \in V$, leads to the maximized instant influence at step $t>0$.

Note that the influence contributions of some nodes may be negative and these nodes should not be selected as white seeds, and thus the optimal solution may have fewer than $k$ seeds. The rules for average influence maximization and long-term influence maximization are patterned in the same way. Therefore, the central task now becomes the computation of the influence contributions of individual nodes. Below, we will introduce our SVIM algorithm, for Signed Voter model Influence Maximization.

\subsection{Short-Term Influence Maximization}

By applying Definition 4.1 and Lemma 4.3, we develop the SVIM-S algorithm to solve the short-term instant and average influence maximization problems, as shown in Algorithm 1.

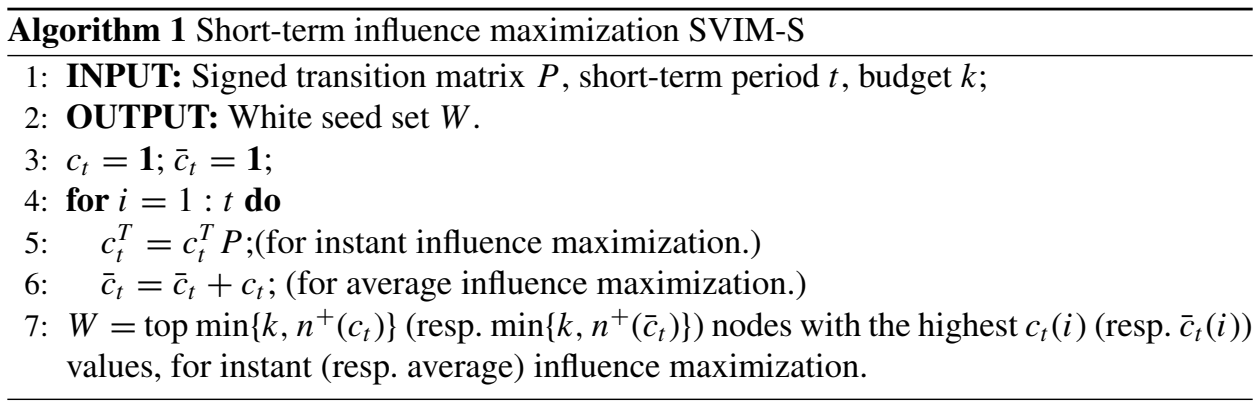

SVIM-S algorithm requires $t$ vector-matrix multiplications, each of which takes $|E|$ times entry-wise multiplication operations. Hence, the total time complexity of SVIM-S is $O(t \cdot|E|)$. Note that when $t$ is sufficiently large (e.g., $t>|V|^{2}$ ), the FOR loop in Algorithm 1 can be modified as follows to reduce the number of FOR Loops to $O(\log t)$, and the total complexity to $O(\log t \cdot|V| \cdot|E|)$.

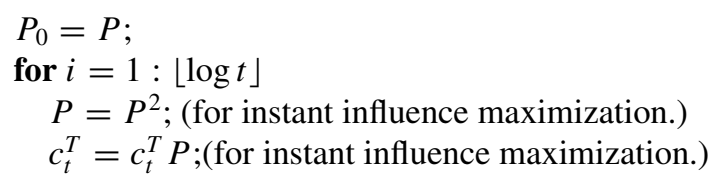


$\bar{c}_{t}=\bar{c}_{t}+c_{t}$; (for average influence maximization.)

for $i=2^{\lfloor\log t\rfloor}+1: t$

$c_{t}^{T}=c_{t}^{T} P_{0}$;(for instant influence maximization.)

$\bar{c}_{t}=\bar{c}_{t}+c_{t}$; (for average influence maximization.)

$P=P^{2}$ in the first loop is performed by matrix multiplication in $O(|E| \cdot|V|+|E|) \approx O(|E|$. $|V|$ ), instead of vector-matrix multiplication in $O(|E|)$. Thus, the overall computation complexity is $O(\log t \cdot|V| \cdot|E|)$. Hence, when $t>\log t \cdot|V|$, the modified Algorithm 1 works better; otherwise, the original Algorithm 1 is better. When $t>|V|^{2}$, we have $t>\log t \cdot|V|$ for any $|V|>1$, thus, the modified Algorithm 1 is better.

\subsection{Long-Term Influence Maximization}

We now study the long-term influence contribution $c$ and introduce the corresponding influence maximization algorithm SVIM-L. We will see that the computation of influence contribution $c$ and seed selection schemes depends on the structural balance and connectedness of the graph. Although seed selection for balanced ergodic digraphs still has intuitive explanations, the computation for weakly connected and disconnected digraphs is more involved and less intuitive.

4.3.1. Case of Ergodic Signed Digraphs. When the signed digraph $G=$ $(V, E, A)$ is ergodic, Lemma 4.4 following, characterizes the long-term influence contributions of node, with respect to various balance structures.

Lemma 4.4. Consider an ergodic signed digraph $G=(V, E, A)$. If $G$ is balanced, with bipartition $S$ and $\bar{S}$, the influence contribution vector $c=(|S|-|\bar{S}|) \hat{\pi}_{S}$. If $G$ is antibalanced or strictly unbalanced, $c=\boldsymbol{0}$.

\section{Proof.}

1. When $G$ is balanced, by Lemma 3.6 and (4.5),

$$
c^{T}=\mathbf{1}^{T} \lim _{t \rightarrow \infty} P^{t}=\mathbf{1}^{T} \hat{\mathbf{1}}_{S} \hat{\pi}_{S}^{T}=(|S|-|\bar{S}|) \hat{\pi}_{S} .
$$

2. When $G$ is strictly unbalanced, again by Lemma 3.6 and (4.5), we have $c^{T}=$ $\mathbf{1}^{T} \lim _{t \rightarrow \infty} P^{t}=\mathbf{0}$.

3. When $G$ is antibalanced, by Lemma 3.6 and (4.4), we have

$$
c^{T}=\mathbf{1}^{T} \frac{\lim _{t \rightarrow \infty} P^{2 t}+\lim _{t \rightarrow \infty} P^{2 t+1}}{2}=\mathbf{0} .
$$

Based on Lemma 4.4, Algorithm 2 summarizes how to compute the long-term influence contribution $c$ on ergodic signed digraphs.

Lemma 4.4 suggests that for ergodic balanced digraphs, we should pick the larger component, e.g., $S$, if $|S|>|\bar{S}|$, and select the top $\min \{k,|S|\}$ nodes from $S$ with the largest stationary distributions as white seeds. Selecting these nodes will guarantee the larger component to become white with a larger probability.

Theorem 3.9 indicates that given an antibalanced digraph $G$, with bipartition $S$ and $\bar{S}$, the long-term dynamic $x_{t}$ oscillates on odd and even steps, and their long-term 


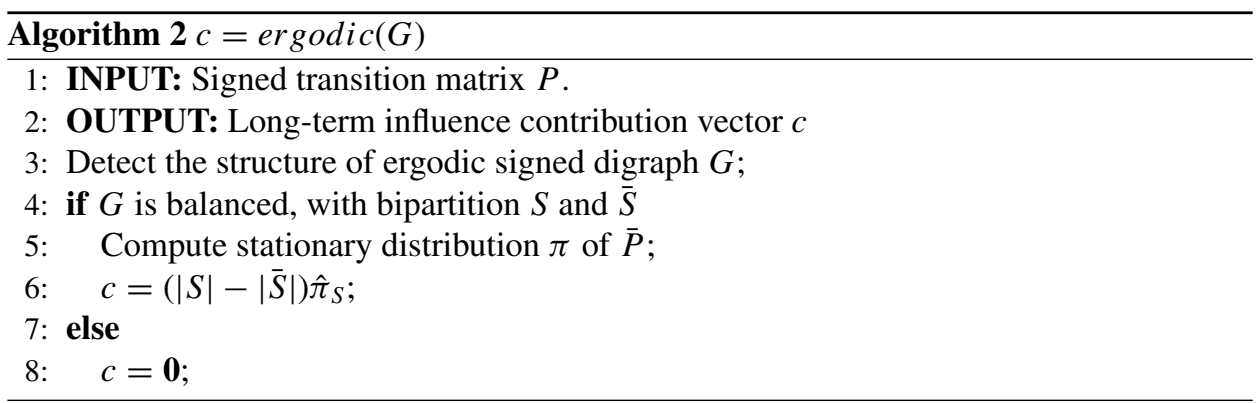

influence contribution is 0 . Define the strength of the oscillation as the difference of the long-term influence between the even vs. odd steps, i.e., $\Delta f_{o, e}=\left|f_{o}\left(x_{0}\right)-f_{e}\left(x_{0}\right)\right| / 2$. We can maximize such strength of the oscillation of the voter model on an antibalanced ergodic digraph by properly choosing the initial white seeds (See Remark 4.5.)

Remark 4.5. In an antibalanced ergodic digraph $G=(V, E, A)$ with the bipartition $S$ and $\bar{S}$ and a budget $k$. Let $W^{\prime}\left(\right.$ resp. $W^{\prime \prime}$ ) denote two initial seed sets, such that $\min \{k,|S|\}$ (resp. $\min \{k,|\bar{S}|\}$ ) nodes are selected with highest stationary distribution $\pi(i)$ 's in $S$ (resp. $\bar{S})$. Then, the optimal $W^{*}$ that maximizes the strength of oscillation $\Delta f_{o, e}$ is

$$
W^{*}:=\underset{W \in\left\{W^{\prime}, W^{\prime \prime}\right\}}{\operatorname{argmax}}\left|\hat{\pi}_{S}^{T}\left(e_{W}-\frac{1}{2} \mathbf{1}\right)\right| .
$$

Proof. From Theorem 3.9, when $t$ becomes sufficiently large, the vector $x$ oscillates at two vectors on odd and even steps, respectively. Rewrite the strength of the oscillation as

$$
\begin{aligned}
\Delta f_{o, e}=\frac{\left|f_{o}\left(x_{0}\right)-f_{e}\left(x_{0}\right)\right|}{2}=\left|\mathbf{1}^{T} \frac{x_{o}\left(x_{0}\right)-x_{e}\left(x_{0}\right)}{2}\right| & =\left|\mathbf{1}^{T} \hat{\mathbf{1}}_{S} \hat{\pi}_{S}^{T}\left(x_{0}-\frac{1}{2} \mathbf{1}\right)\right| \\
& =|| S|-| \bar{S}|| \cdot\left|\hat{\pi}_{S}^{T}\left(x_{0}-\frac{1}{2} \mathbf{1}\right)\right| .
\end{aligned}
$$

Let $W$ be the initial seed set, then the oscillation strength maximization is formulated as

$$
\begin{aligned}
& \max _{|W| \leq k}|| S|-| \bar{S}|| \cdot\left|\hat{\pi}_{S}^{T}\left(e_{W}-\frac{1}{2} \mathbf{1}\right)\right| \\
& \quad=|| S|-| \bar{S}|| \cdot \max \left\{\max _{|W| \leq k}\left\{\hat{\pi}_{S}^{T} e_{W}\right\}-\frac{1}{2} \hat{\pi}_{S}^{T} \mathbf{1}, \max _{|W| \leq k}\left\{-\hat{\pi}_{S}^{T} e_{W}\right\}+\frac{1}{2} \hat{\pi}_{S}^{T} \mathbf{1}\right\},
\end{aligned}
$$

which contains two subproblems, i.e., $\max _{|W| \leq k}\left\{\hat{\pi}_{S}^{T} e_{W}\right\}$ and $\max _{|W| \leq k}\left\{-\hat{\pi}_{S}^{T} e_{W}\right\}$. The first maximization problem can be rewritten as

$$
\max _{|W| \leq k}\left\{\hat{\pi}_{S}^{T} e_{W}\right\}=\max _{|W| \leq k}\left(\sum_{i \in S} \pi(i) e_{W}(i)-\sum_{j \in \bar{S}} \pi(i) e_{W}(j)\right) .
$$


Thus, let $W^{\prime}$ denote the optimal solution to the problem in (4.8), which is obtained by choosing $\min \{k,|S|\}$ seeds with highest $\pi(i)$ 's from $S$. Similarly, choosing $\min \{k,|\bar{S}|\}$ nodes with the highest $\pi(i)$ 's from $\bar{S}$ yields the optimal solution, denoted by $W^{\prime \prime}$, to the second maximization problem $\max _{|W| \leq k}\left\{-\hat{\pi}_{S}^{T} e_{W}\right\}$. The optimal $W$ to the problem in (4.7) that maximizes the oscillation strength is the one in $\left\{W^{\prime}, W^{\prime \prime}\right\}$, with higher $\left|\hat{\pi}_{S}^{T}\left(e_{W}-\frac{1}{2} \mathbf{1}\right)\right|$, which completes the proof of (4.6).

4.3.2. Case of Weakly Connected Signed Digraphs. We first consider a weakly connected signed $G$, which has a single ergodic sink component $G_{Z}$ with only incoming edges from the remaining nodes $X=V \backslash Z$.

Lemma 4.6. Consider a weakly connected digraph $G=(V, E, A)$ with a single ergodic sink component $G_{Z}$. If $G_{Z}$ is balanced, with partition $S_{Z}$ and $\overline{S_{Z}}$, the long-term influence contribution vector $c^{T}=\left[c_{X}^{T}, c_{Z}^{T}\right]$, where $c_{X}=\boldsymbol{0}_{X}$ and $c_{Z}=\left(\boldsymbol{1}_{X}^{T} u_{b}+\left|S_{Z}\right|-\left|\bar{S}_{Z}\right|\right) \hat{\pi}_{Z, S_{Z}}$. If $G$ is antibalanced or strictly unbalanced, $c=0$.

\section{Proof.}

1. When $G_{Z}$ is balanced, by Lemma $3.7, c_{X}=\mathbf{0}_{X}$, and

$$
c_{Z}^{T}=\left(\mathbf{1}_{X}^{T} u_{b}+\mathbf{1}_{Z}^{T} \hat{\mathbf{1}}_{Z, S_{Z}}\right) \hat{\pi}_{Z, S_{Z}}^{T}=\left(\mathbf{1}_{X}^{T} u_{b}+\left|S_{Z}\right|-\left|\bar{S}_{Z}\right|\right) \hat{\pi}_{Z, S_{Z}}^{T}
$$

2. When $G_{Z}$ is strictly unbalanced, $c^{T}=\mathbf{1}^{T} \lim _{t \rightarrow \infty} P^{t}=\mathbf{0}$

3. When $G_{Z}$ is antibalanced, by Lemma 3.7 the limits of odd and even subsequences of $P^{t}$ cancel out, thus $c=\mathbf{0}$.

Lemma 4.6 indicates that influence contribution of the balanced ergodic sink component is more complicated than that of the balanced ergodic digraph. This is because the sink component affects the colors of the nonsink component in a complicated way depending on how nonsink and sink components are connected. Therefore, the optimal seed selection depends on the calculation of the influence contributions of each sink node, and is not as intuitive as that for the ergodic digraph case.

Theorem 3.11 shows that in a weakly connected signed digraph $G$, with single antibalanced sink component $G_{Z}$, the long-term influence $f\left(x_{0}\right)$ oscillates on odd and even steps, and the average is $|V| / 2$, which is invariant to the initial seed selection. Similar to Remark 4.5 , we can maximize the oscillation strength by properly selecting initial seeds, i.e.,

$$
\begin{aligned}
W^{*} & =\underset{|W| \leq k}{\operatorname{argmax}}\left|f_{e}\left(e_{W}\right)-f_{o}\left(e_{W}\right)\right| / 2 \\
& =\underset{|W| \leq k}{\operatorname{argmax}}\left|\left(\mathbf{1}_{X}^{T} u_{u} \hat{\pi}_{Z, S_{Z}}^{T}+\mathbf{1}_{Z}^{T} \hat{\mathbf{1}}_{Z, S_{Z}} \hat{\pi}_{Z, S_{Z}}^{T}\right)\left(e_{W Z}-\frac{1}{2} \mathbf{1}_{Z}\right)\right| \\
& =\left|\mathbf{1}_{X}^{T} u_{u}+\right| S_{Z}|-| \bar{S}_{Z}|| \cdot \underset{|W| \leq k}{\operatorname{argmax}}\left|\hat{\pi}_{Z, S_{Z}}^{T}\left(e_{W Z}-\frac{1}{2} \mathbf{1}_{Z}\right)\right|
\end{aligned}
$$


where the maximization objective is independent from $x_{0 X}$, thus oscillation strength maximization problem objective in (4.9) for $G$ is identical to that in Remark 4.5. Hence, Remark 4.5 also applies here.

Using (3.15), Lemma 4.4 and Lemma 4.6 can be readily extended to the case with more than one ergodic sink component and disconnected digraphs. Algorithm 3 summarizes how to compute the node influence contributions of weakly connected signed digraphs. Note that, by our assumption, we consider all sink components to be ergodic.

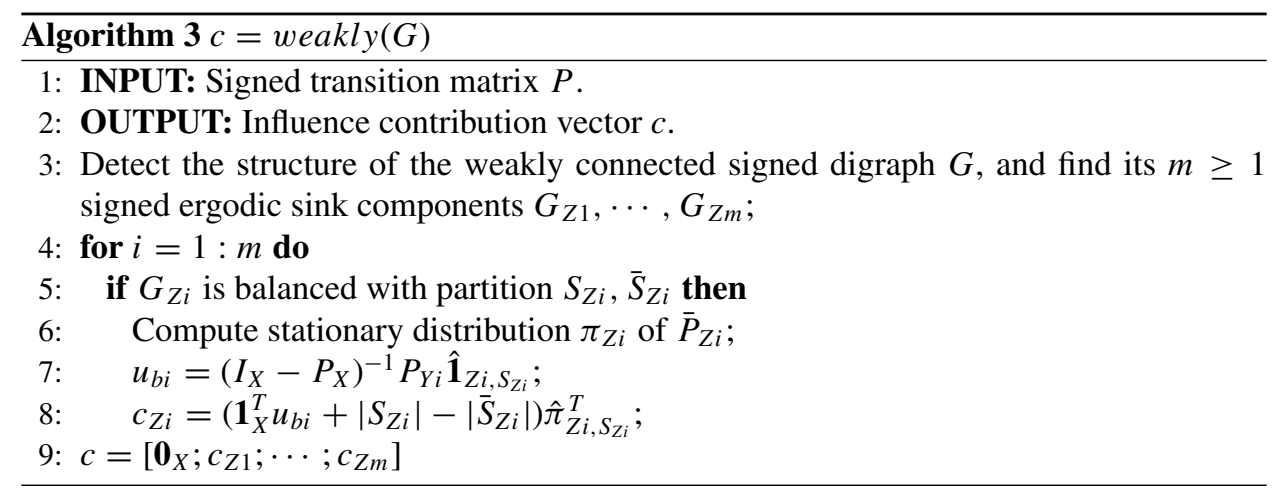

4.3.3. General Case and SVIM-L Algorithm. Given the above systematic analysis, we are now in a position to summarize and introduce our SVIM-L algorithm, which solves the long-term voter model influence maximization problem for general aperiodic signed digraphs.

In general, a signed digraph consists $m \geq 1$ disconnected components, within each of which the node influence contribution follows Lemma 4.6. The long-term signed voter model influence maximization (SVIM-L) algorithm is constructed in Algorithm 4.

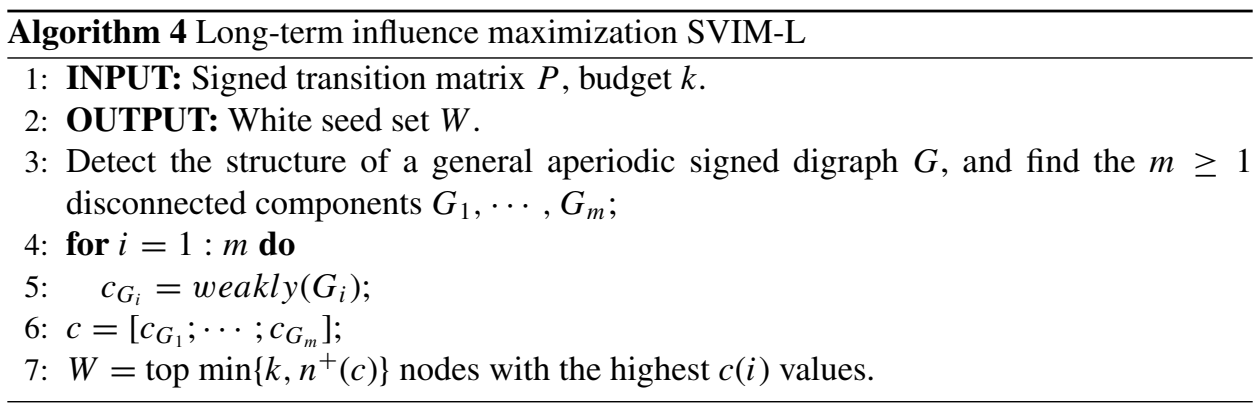

4.3.4. Complexity Analysis. We consider $G=(V, E, A)$ to be weakly connected, because the disconnected graph case can be treated independently for each connected component for the time complexity. The SVIM-L algorithm consists of two parts. The first part extracts the connectivity and balance structure of the graph, which can be done using depth-first search with complexity $O(|E|)$. The second part uses Algorithm 3 to compute influence contributions of balanced ergodic sink components. The dominant computations are on the stationary distribution $\pi_{Z i}$ 's and $\left(I_{X}-P_{X}\right)^{-1}$, which can be done by solving a linear equation system [41] and matrix inverse in $O\left(\left|Z_{i}\right|^{3}\right)$ and $O\left(n_{X}^{3}\right)$, respectively, where $n_{X}=|X|$. Let $b$ be the number of balanced sink components in $G, n_{Z}$ be 
the number of nodes in the largest balanced sink component. Thus SVIM-L can be done in $O\left(b n_{Z}^{3}+n_{X}^{3}\right)$ time. Alternatively, we can use the iterative method for computing both $\pi_{Z i}$ 's and $\mathbf{1}_{X}^{T}\left(I_{X}-P_{X}\right)^{-1}$, if the largest convergence time $t_{C}$ of $P_{Z i}^{t}$ 's and $P_{X}^{t}$ is small. (Note that the convergence time of ergodic digraphs could be exponentially large in general, as illustrated by an example in Appendix C). In this case, each iteration step involves vectormatrix multiplication and can be done in $O\left(m_{B}\right)$ time, where $m_{B}$ is the number of edges of the induced subgraph $G_{B}$ consisting of all nodes in the balanced sink components and $X$. Note that $m_{B}$ and $t_{C}$ are only related to subgraph $G_{B}$, which could be significantly smaller than $G$, and thus $O\left(t_{C} m_{B}\right)$ could be much smaller than the time of naive iterations on the entire graph. Overall SVIM-L can be done in $O\left(|E|+\min \left(b n_{Z}^{3}+n_{X}^{3}, t_{C} m_{B}\right)\right)$ time.

\section{EVALUATION}

In this section, we first use both synthetic datasets and real social network datasets to demonstrate the efficacy of our short-term and long-term seed selection schemes by comparing the performances with four baseline heuristics. Then, we evaluate how much the short-term and long-term influence can be improved by taking the edge signs into consideration.

\subsection{Performance Comparison with Baseline Heuristics}

For different scenarios, we compare our SVIM-L and SVIM-S algorithms with four heuristics, i.e., (1) selecting seed nodes with the highest weighted outgoing degrees (denoted by $d^{+}+d^{-}$in the figures), (2) highest weighted outgoing positive degrees (denoted by $d^{+}$), (3) highest differences between weighted outgoing positive and negative degrees (denoted by $d^{+}-d^{-}$), and (4) randomly selecting seed nodes (denoted by "Rand"), where, in our evaluations, we run random seed selection 1000 times, and compare the average number of white nodes between our algorithm and other heuristics. Our evaluation results demonstrate that our seed selection scheme can increase up to $72 \%$ long-term influence, and $145 \%$ short-term influence over other heuristics.

\subsection{Synthetic Datasets}

In this part, we generate synthetic datasets with different structures to validate our theoretical results.

5.2.1. Dataset Generation Model. We generate six types of signed digraphs, including balanced ergodic digraphs, antibalanced ergodic digraphs, strictly unbalanced ergodic digraphs, weakly connected signed digraphs, disconnected signed digraphs with ergodic components, and disconnected signed digraphs with weakly connected components (WCCs). All edges have unit weights. The following are graph configuration details.

We first create an unsigned ergodic digraph $\bar{G}$ with 9500 nodes, which has two ergodic components $\bar{G}_{A}$ and $\bar{G}_{B}$, with [3000, 6500] nodes and [3000, 6500] $\times 8$ random directed edges, respectively. Moreover, there are $3000 \times 8$ random directed edges across $\bar{G}_{A}$ and $\bar{G}_{B}$. Ergodicity is checked through a simple connectivity and aperiodicity check. Given $\bar{G}$, a balanced digraph is obtained by assigning all edges within $\bar{G}_{A}$ and $\bar{G}_{B}$ with positive signs, and those across them with negative signs. Then, an antibalanced digraph is generated by negating all edge signs of the balanced ergodic digraph. To generate a strictly 


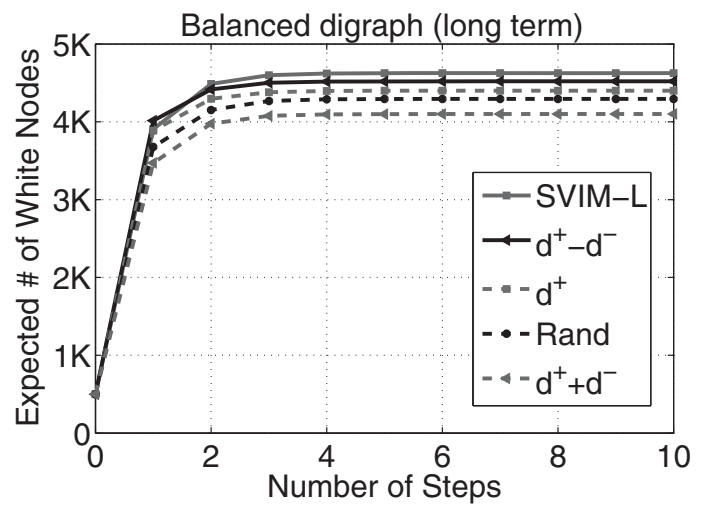

Figure $1 G$ is balanced.

unbalanced digraph, we randomly assign edge signs to all edges in $\bar{G}$ and make sure that there does not exist a balanced or antibalanced bipartition.

Moreover, we generated a disconnected signed digraph and a weakly connected signed digraph for our study. We first generate five ergodic unsigned digraphs, $\bar{G}_{1}, \cdots, \bar{G}_{5}$ with $[500,200,800,300,2700]$ nodes and $[500,200,800,300,2700] \times 8$ edges, respectively. Then, we group $G_{23}=\left(G_{2}, G_{3}\right)$ and $G_{45}=\left(G_{4}, G_{5}\right)$ to form two ergodic balanced digraphs, and generate a strictly unbalanced ergodic digraph $G_{1}$ by randomly assigning signs to edges in $\bar{G}_{1}$. Three disconnected components $G_{1}, G_{23}, G_{45}$ together form a disconnected signed digraph. To form a weakly connected signed digraph, we place, in total, 3000 random direct edges from $G_{1}$ to the balanced ergodic components $G_{23}$ and $G_{45}$, where the nodes in subgraph $G_{1}$ have only outgoing edges to $G_{23}$ and $G_{45}$. Moreover, we combine the above generated balanced ergodic digraph and the weakly connected signed digraph, forming a larger disconnected signed digraph, with the weakly connected signed digraph as a component.

Figure 1-Figure 6 present the evaluation results for one set of digraphs, in which we observe that all digraphs we randomly generated exhibit consistent results. Our tests are conducted using MATLAB on a standard PC server.

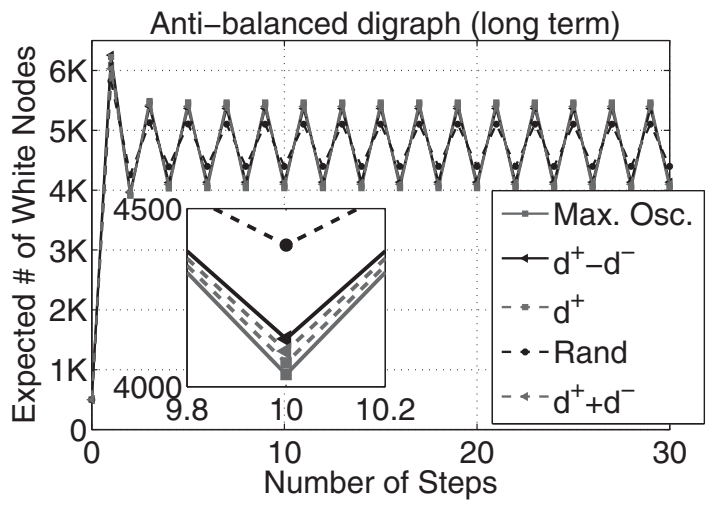

Figure $2 G$ is antibalanced. 


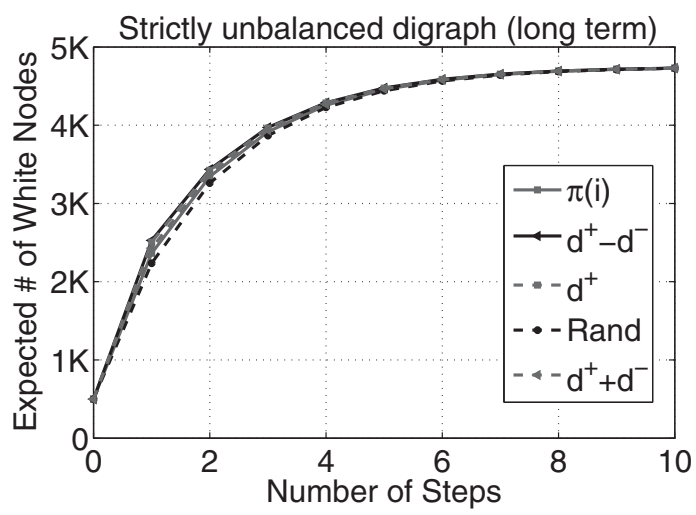

Figure $3 G$ is strictly unbalanced.

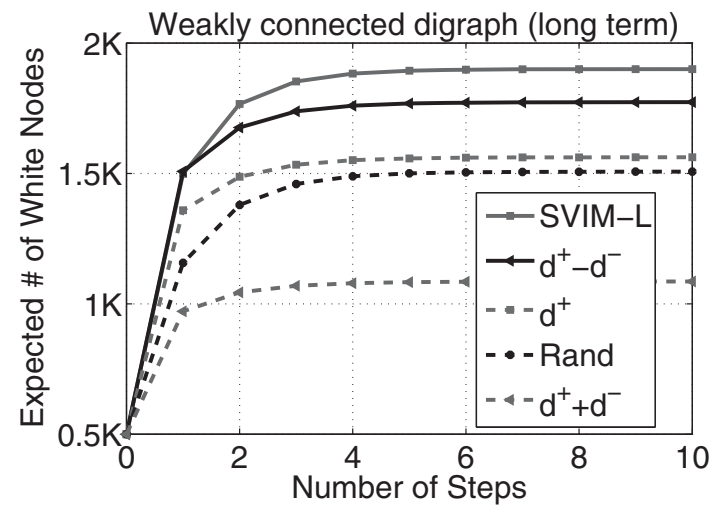

Figure $4 G$ is weakly connected.

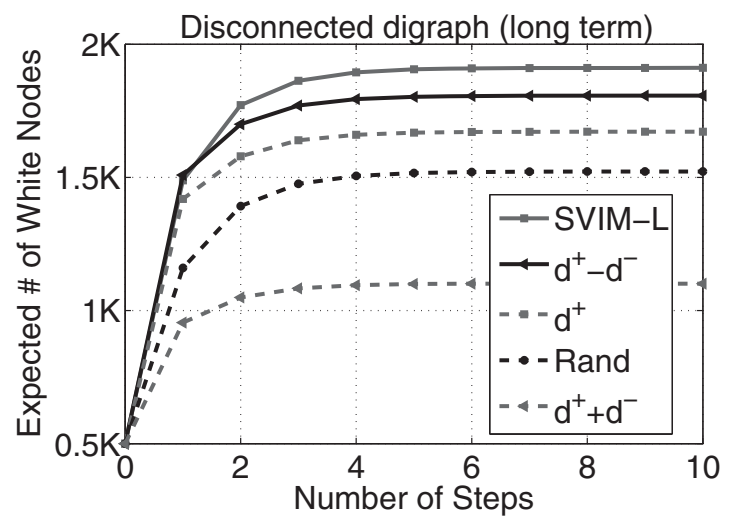

Figure $5 G$ is disconnected. 


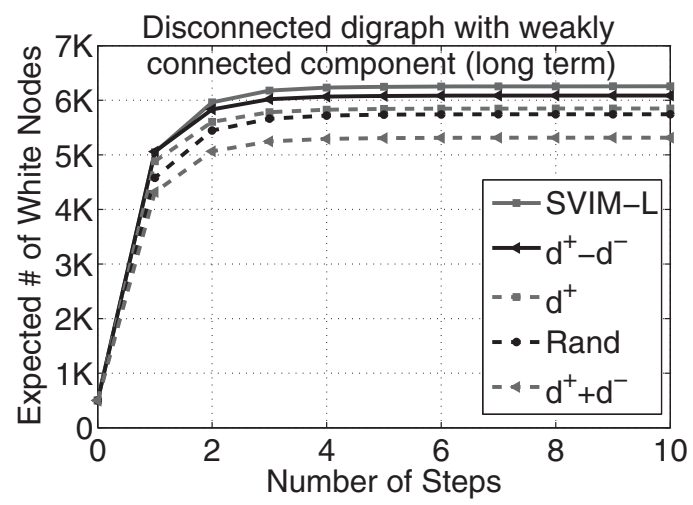

Figure $6 G$ is disconnected with WCC.

5.2.2. Long-Term Influence Maximization. In the evaluations, we set the influence budget as $k=500$ and compare the average numbers of white nodes over steps between our algorithm and other heuristics. Figure 1 shows that in the balanced ergodic digraph, the SVIM-L algorithm, achieves the highest long-term influence over other heuristics. When applying a heuristic seed selection scheme, denoted by $\mathrm{H}, f_{t}^{\mathrm{H}}$ represents the number of white nodes at step $t(\geq 1)$. Similarly, denote $f_{t}^{\text {SVIM }}$ as the number of white nodes at step $t(\geq 1)$ for the SVIM algorithm. We consider $\Delta f_{t}(\mathrm{SVIM}, \mathrm{H})=\left(f_{t}^{\mathrm{SVIM}}-f_{t}^{\mathrm{H}}\right) / f_{t}^{\mathrm{H}}$ as the influence increase of SVIM over the heuristic algorithm $\mathrm{H}$ at step $t$. The maximum influence increase is the maximum $\Delta f_{t}(\mathrm{SVIM}, \cdot)$ among all steps $(t \geq 1)$ and all heuristics. Hence, in Figure 1, we see that our SVIM-L algorithm outperforms all other heuristics. Especially, a maximum of $14 \%$ influence increase is observed for $t \geq 4$ with $4.68 k$ and $4.1 k$ white nodes for SVIM-L and random selection scheme, respectively. In the rest of this section, we will use the maximum influence increase as a metric to illustrate the efficacy of our SVIM algorithm. Figure 2 shows the clear oscillating behavior on the antibalanced ergodic digraph, and the average influence is the same for all algorithms. The inset shows that our algorithm (denoted as "Max. Osc.") indeed provides the largest oscillation. Figure 3 shows the results in the strictly unbalanced graph case, wherein the long-term influences

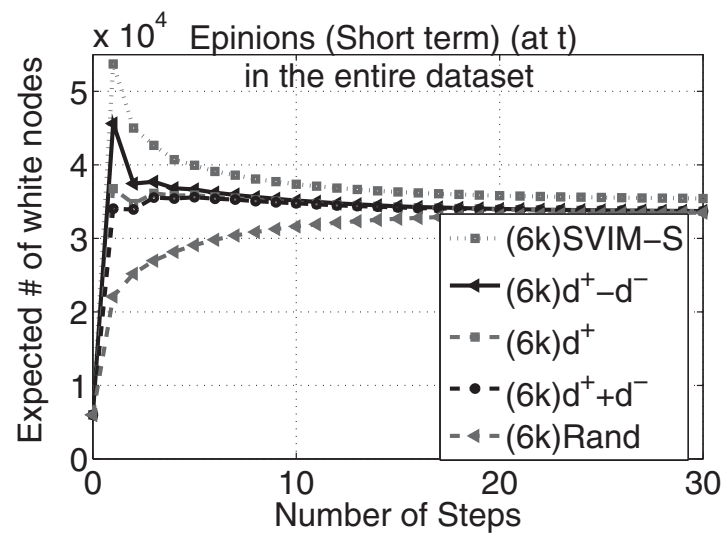

Figure 7 Instant influence in Epinions data with $k=6 \mathrm{k}$. 


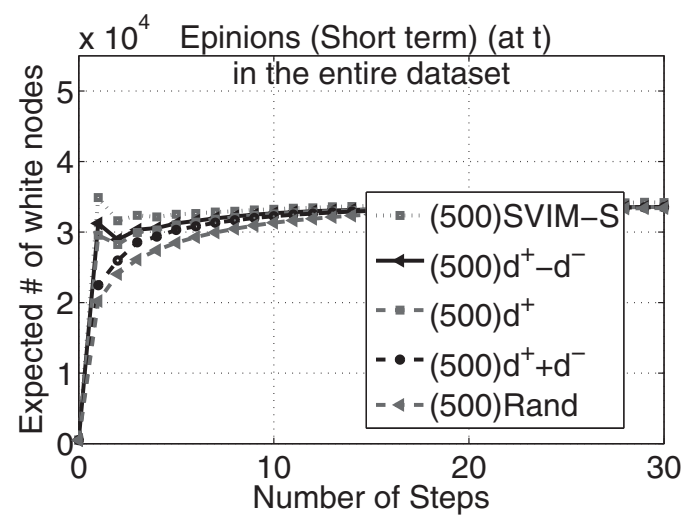

Figure 8 Instant influence in Epinions data with $k=500$.

of all algorithms converge to $4750=|V| / 2$, which matches Theorem 3.9. Figures 4 and 5 show that the SVIM-L algorithm performs the best, and it generates 5.6\% - 72\% long-term influence increases after the sixth step over other heuristics in the weakly connected signed digraph and the disconnected signed digraph. Figure 6 shows that in a more general signed digraph, which consists of a weakly connected signed component and a balanced ergodic component, the SVIM-L algorithm outperforms all other heuristics with up to $17 \%$ more long-term influence, which occurs for $t \geq 4$. In general, we see that for weakly connected and disconnected digraphs, SVIM-L has larger winning margins over all other heuristics than the balanced ergodic digraphs (Figures 4-6 vs. Figure 1). We attribute this to our accurate computation of influence contribution in the more involved weakly connected and disconnected digraph cases. Moreover, in all cases, the dynamics converge very fast, i.e., in only a few steps, which indicates that the convergence times of the voter model on these random graphs are very small.

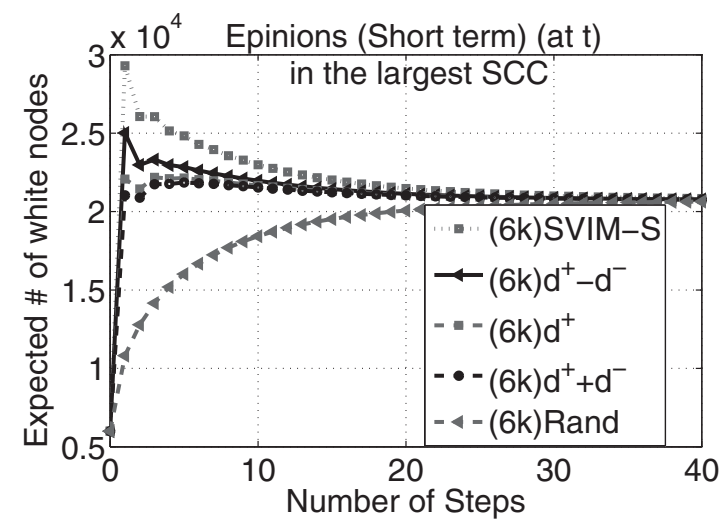

Figure 9 Instant influence in SCC with $k=6 \mathrm{k}$. 


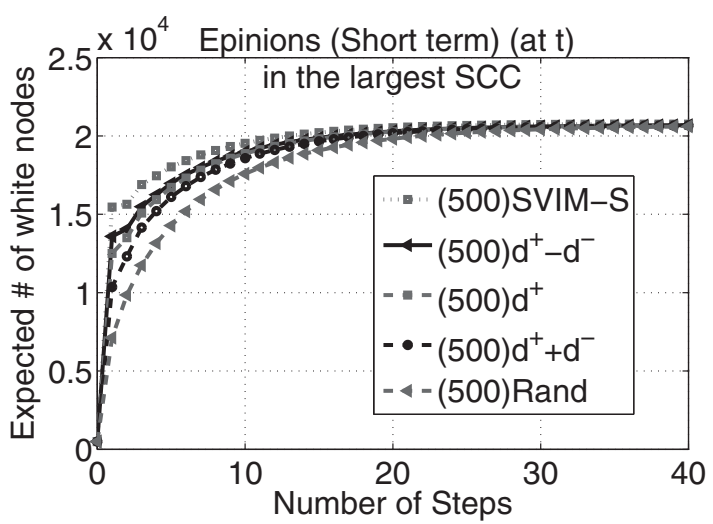

Figure 10 Instant influence in SCC with $k=500$.

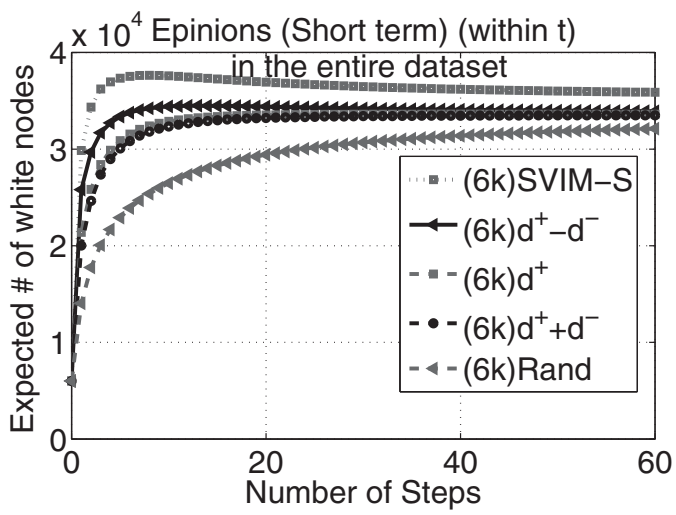

Figure 11 Average influence in Epinions data with $k=6 \mathrm{k}$.

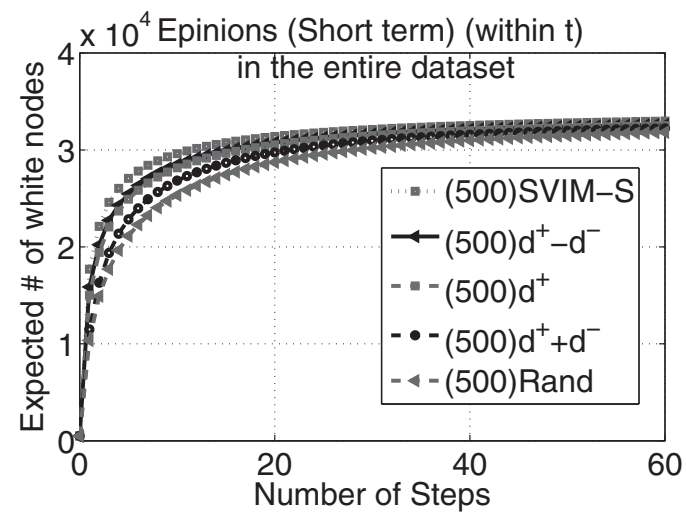

Figure 12 Average influence in Epinions data with $k=500$. 


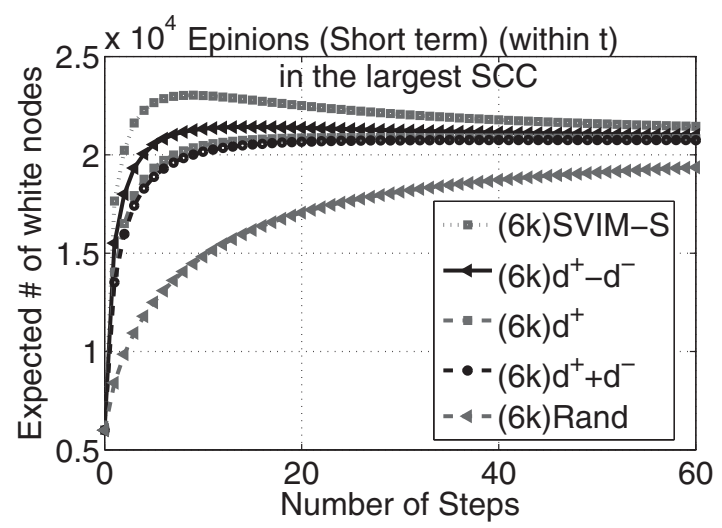

Figure 13 Average influence in SCC with $k=6 \mathrm{k}$.

\subsection{Real Datasets}

We conduct extensive simulations using real datasets, such as Epinions and Slashdot datasets, to validate our theoretical results and evaluate the performance of our SVIM algorithm.

5.3.1. Epinions Dataset. Epinions [15] is a consumer review online social site, where users can write reviews to various items and vote for or against other users. The signed digraph is formed with positive or negative directed edge $(u, v)$ meaning that $u$ trusts or distrusts $v$. The statistics are shown in Table II. We compare our short-term SVIM-S algorithm with four heuristics, i.e., $d^{+}+d^{-}, d^{+}, d^{+}-d^{-}$and random seed selection, on the entire Epinions digraph as well as the largest strongly connected component (SCC).

Our tests are conducted on both the Epinions dataset and its largest SCC, where the largest SCC is ergodic and strictly unbalanced. We first look at the comparison of instant influence maximization ( $A t$ step $t$ ) among various seed selection schemes. Figures 7-10 shows the expected maximum instant influence at each step by different methods. Note that since the initial seeds selected by the SVIM-S algorithm hinge on $t$, the values on the curve of our selection scheme are associated with different optimal initial seed sets. To the

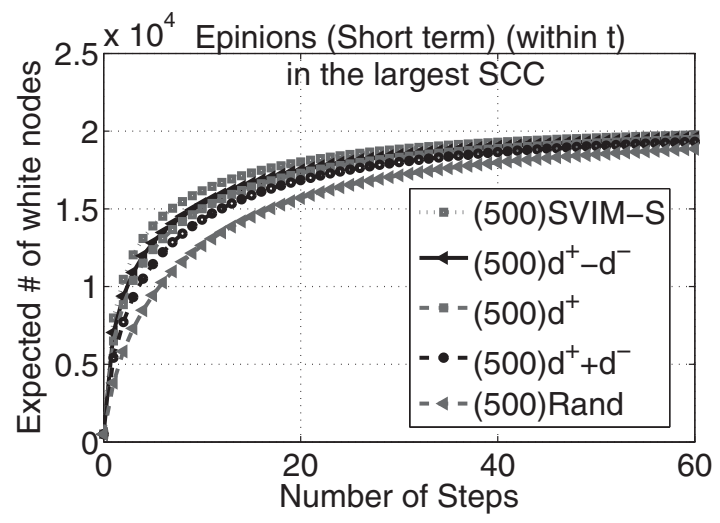

Figure 14 Average influence in SCC with $k=500$. 


\begin{tabular}{|lrr|}
\hline Statistics & Epinions & Slashdot \\
\hline \hline \# of nodes & 131580 & 77350 \\
\# of edges & 840799 & 516575 \\
\# of positive edges & 717129 & 396378 \\
\# of negative edges & 123670 & 120197 \\
\# of nodes in largest SCC & 41441 & 26996 \\
\# of edges in largest SCC & 693507 & 337351 \\
\# of positive edges in largest SCC & 614314 & 259891 \\
\# of negative edges in largest SCC & 79193 & 77460 \\
\# of strongly connected components & 88361 & 49209 \\
\hline
\end{tabular}

Table II Statistics of Epinions and Slashdot datasets.

contrary, the seed selections of other heuristics are independent to $t$, thus the corresponding curves represent the same initial seed sets. We choose the budget as 500 and 6000 in our evaluations, i.e., selecting at maximum 500 or 6000 initial white seeds. From Figures 7-10, the SVIM-S algorithm consistently performs better, and in some cases, e.g., Figure 9, it generates 16\%-145\% more influence than other heuristics at Step 1.

Next we compare the seed selection schemes for maximizing the average influence within the first $t$ steps. Figures 11-14 show the expected maximum average influence within the first $t$ steps by different methods. Again, the values on the curve of the SVIM$\mathrm{S}$ algorithm are associated with different initial seed sets. Figures 11-14 show that with different budgets, i.e., 500 and 6000 seeds, the SVIM-S algorithm performs better than all other heuristics, where, in Figure 13, a maximum of $64 \%$ more influence is achieved at $t=8$. Moreover, in all these figures, we observe that our seed selection scheme results in the highest long-term influence over other heuristics.

Moreover, from Figures 7-14, we observe that as $t$ increases, the influences (i.e., the expected number of white nodes) for SVIM-S and all heuristics, except for the random seed selection schedule, increase for small $t$ 's and then decrease and converge to the stationary state. In contrast, from Figures 1-6, the influence increases monotonically with $t$. This happens because the Epinions dataset (as well as many real-network datasets) has a large portion (around 80\%) of nodes in the nonsink components, where, to maximize the long-term influence, only nodes in sink components should be selected, which governs the

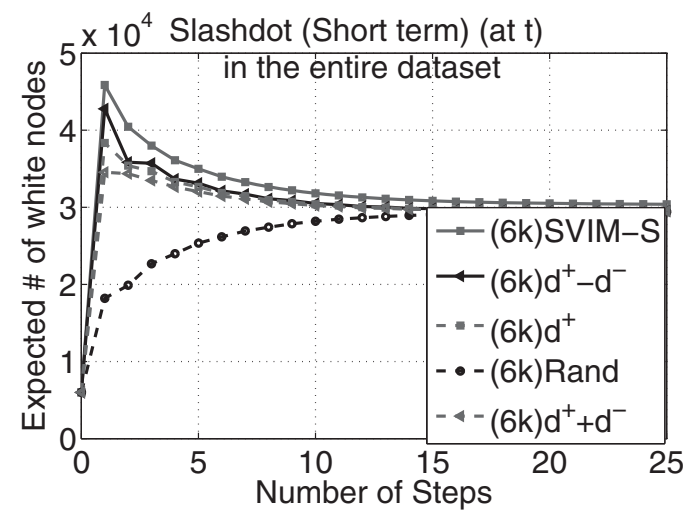

Figure 15 Instant influence in Slashdot data with $k=6 \mathrm{k}$. 


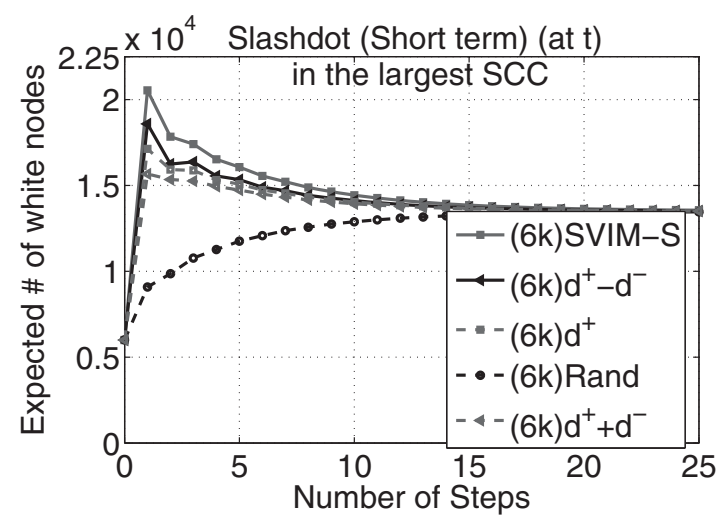

Figure 16 Instant influence in Slashdot SCC with $k=6 \mathrm{k}$.

long-term influence dynamics of the whole graph; that is, sink nodes have higher long-term influence contributions. However, for short-term influence maximization, nodes with higher chances to influence more nodes in a few steps generally have a large number of incoming links, which are able to influence a large number of nodes in either sink or nonsink components in a short period of time. Hence, in signed digraphs with large nonsink components, given a sufficiently large budget, the short-term influence can definitely outnumber the long-term influence. Our evaluations confirm this explanation. This interesting observation also leads to a problem: given a budget $k$, how to find the optimal time step $t$ that generates the largest influence among all possible $t$ 's. We leave this problem as our future work.

5.3.2. Slashdot Dataset. Slashdot [39] provides a discussion forum on various technology-related topics, where members can submit their stories, and comment on other members' stories. Its Slashdot Zoo feature allows members to tag each other as friends or foes, which in turn forms a signed online social network. The network was collected on November 6, 2008 [25] and the statistics are shown in Table II.

We evaluate instant influence and average influence of our SVIM-S algorithm on the entire slashdot dataset and its largest strongly connected component, respectively. Our results for $k=6000$ are presented in Figures 15-18, which show that our SVIM-S algorithm performs the best among all methods tested, especially in the early steps. When changing the budget $k$, similar results were obtained, where we omitted them here for brevity.

Moreover, the convergence times for both real-world datasets are fast, in a few tens of steps, indicating good connectivity and fast mixing property of real-world networks. In summary, our evaluation results on both synthetic and real-world networks validate our theoretical results and demonstrate that our SVIM algorithms for both short term and long term are indeed the best, and often have significant winning margins.

\subsection{The Impacts of Signed Information}

Unlike Epinions and Slashdot, many online social networks such as Twitter are simply represented by unsigned directed graphs, where friends and foe relationships are not explicitly represented on edges. Without edge signs, two types of information may be misrepresented or underrepresented: (1) one might follow foes for tracking purposes, but this link might be misinterpreted as friend or trust relationship; and (2) one might not 


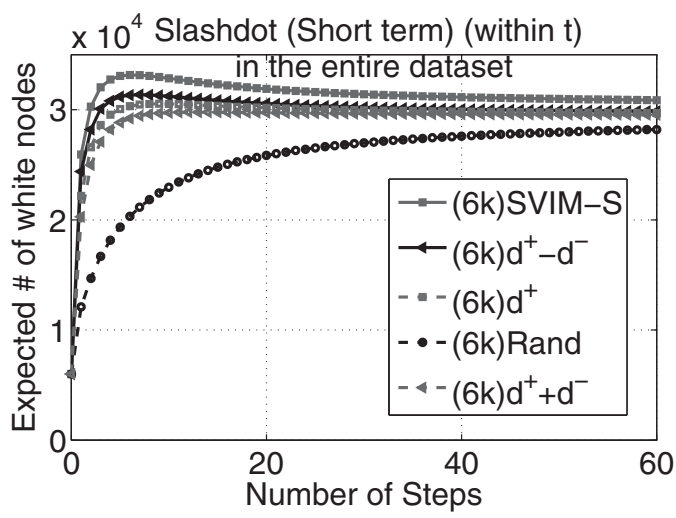

Figure 17 Average influence in Slashdot data with $k=6 \mathrm{k}$.

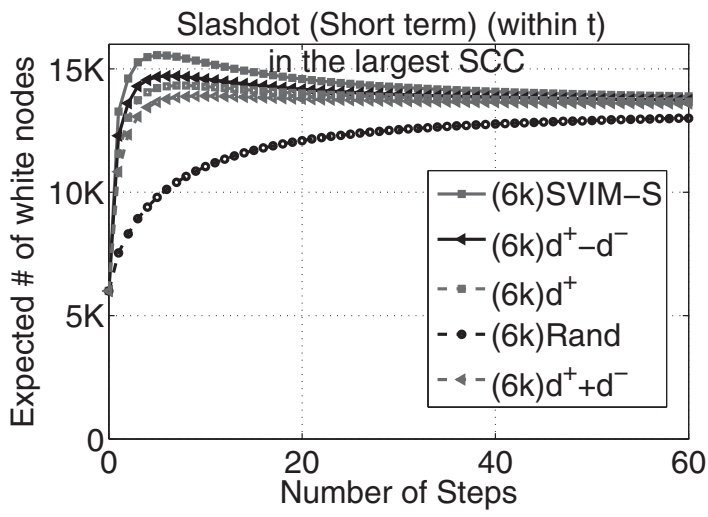

Figure 18 Average influence in Slashdot SCC with $k=6 \mathrm{k}$.

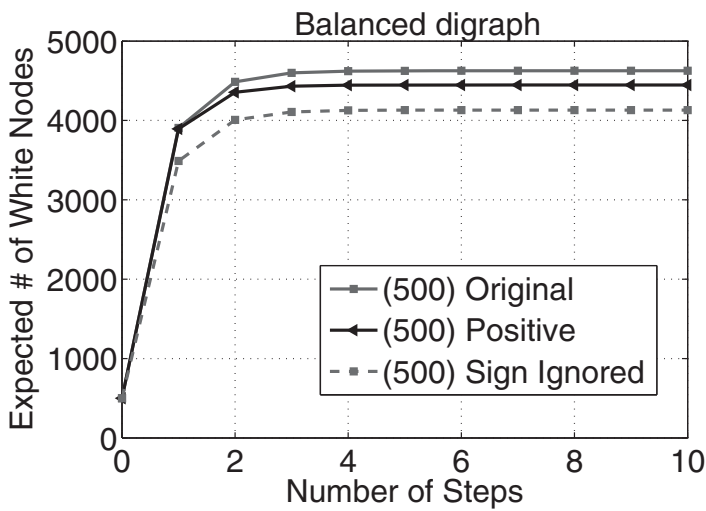

Figure 19 Synthetic balanced digraph. 


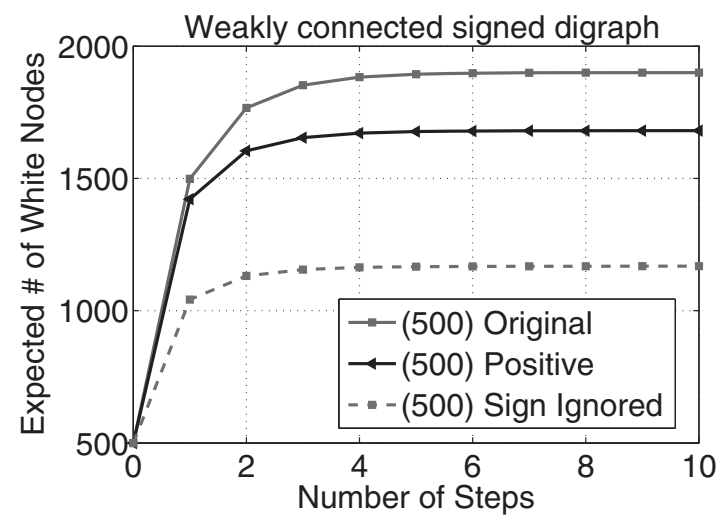

Figure 20 Synthetic weakly connected digraph.

follow foes publicly in order to avoid being noticed, but the foes may still generate negative influence. In this section, we investigate how much influence gain can be obtained by taking the edge signs into consideration, thus illustrating the significance of utilizing both friend and foe relationships in influence maximization.

Taking the synthetic networks and Epinions dataset (used in Section 5.3) as examples, we apply our SVIM algorithm to compute the optimal initial seed sets in the original signed digraphs, and two types of "sign-missing" scenarios, i.e., the unsigned digraphs with only original positive edges (denoted by "Positive" graphs) and with all edges labeled by the same signs (denoted by "Sign ignored" graphs). Then, we examine the performances of those three initial seed sets in original signed digraphs.

Figures 19-22 show the evaluation results, wherein the seed sets obtained by considering edge signs perform consistently better than those using unsigned graphs. In synthetic networks, we observed 5\%-16\% more influence in the balanced digraph for $t \geq 6$ (see Figure 19), and $11.7 \%-58 \%$ more influence in the weakly connected digraph for $t \geq 6$ (see Figure 20). Moreover, in Epinions dataset from Figures 21-22, there is no impact on the long-term influence, since the underlying graphs are strictly unbalanced. However, in short term, the results demonstrate that taking edge signs into consideration always performs

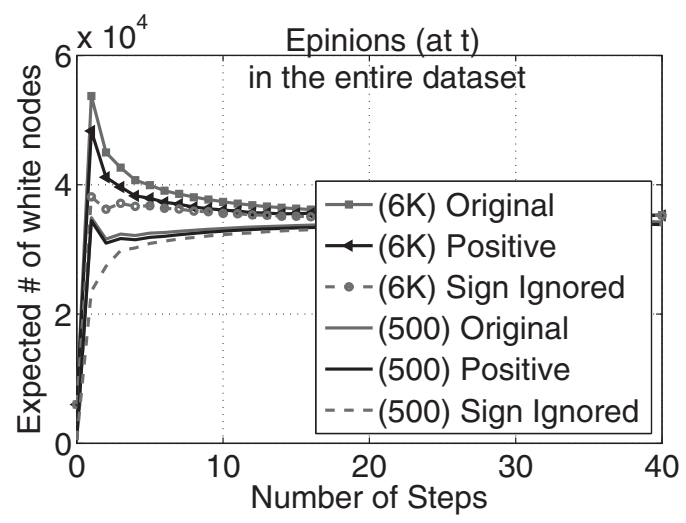

Figure 21 Epinions (the entire dataset). 


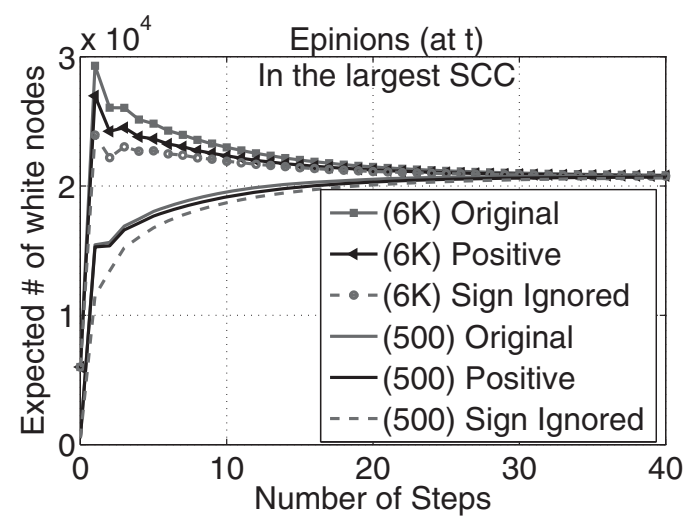

Figure 22 Epinions (the largest SCC).

better, which generates at maximum of $38 \%$ and $21 \%$ more influence for the entire dataset (see Figure 21) and the largest SCC (see Figure 22), respectively. Both maximums occur at Step 1 . These results clearly demonstrate the necessity of utilizing sign information in influence maximization.

\section{CONCLUSION}

In this article, we propose and study voter model dynamics on signed digraphs, and apply it to solve the influence maximization problem. We provide rigorous mathematical analysis to completely characterize the short-term and long-term dynamics and provide efficient algorithms to solve both short-term and long-term influence maximization problems. Extensive simulation results on both synthetic and real-world graphs demonstrate the efficacy of our signed voter model influence maximization (SVIM) algorithms. We also identify a class of antibalanced digraphs, which is not covered in the social balance theory before, and which exhibits oscillating steady state behavior.

There exist several open problems and future directions. One open problem is the convergence time of voter model dynamics on signed digraphs. For balanced and antibalanced ergodic digraphs, our results show that their convergence times are the same as the corresponding unsigned digraphs. For strictly unbalanced ergodic digraphs and more general weakly connected signed digraphs, the problem is quite open. A future direction is to study influence diffusion in signed networks under other models, such as the voter model with a background color, the independent cascade model, and the linear threshold model. Moreover, we are interested in studying the centrality measures of signed digraphs, such as random walk betweenness and shortest path betweenness, originally defined for undirected unsigned graphs [33,34].

\section{ACKNOWLEDGMENTS}

We would like to thank Christian Borgs and Jennifer T. Chayes for pointing out the relations between the signed digraph voter model and concepts in physics, such as the Ising model and Gauge transformations. We also thank Zhenming Liu for many useful 
discussions on this work. This work was mostly done while the first author was working as full-time intern at Microsoft Research Asia.

\section{FUNDING}

Yanhua Li and Zhi-Li Zhang were supported in part by DoD ARO MURI Award W911NF-12-1-0385, DTRA grant HDTRA1-09-1-0050, and NSF grants CNS-1017092, CNS-10171647, and CNS-1117536.

\section{APPENDIX A. PROPERTIES OF ERGODIC DIGRAPHS}

Proposition A.1. Let $G=(V, E, A)$ be an ergodic digraph. For any nodes $i, j \in V$, there exist two paths from $i$ to $j$ with even and odd length, respectively.

Proof. Suppose, for a contradiction, that all paths from $i$ to $j$ have even lengths. This implies that all cycles passing through $i$ must be of even length; otherwise, we could follow node $i$ 's odd-length cycle followed by the even-length path from $i$ to $j$, making the entire path from $i$ to $j$ odd. Now we can consider any cycle $C_{r}$ in $G$, not necessarily passing $i$. We claim that $C_{r}$ must have even length. In fact, we can pick any node $u$ on $C_{r}$, and construct a path from $i$ to $j$ with the following segments: $R_{1}$ from $i$ to $u, C_{r}, R_{2}$ from $u$ back to $i$, and $R_{3}$ from $i$ to $j$. Because we know that $R_{1}+R_{2}$ has even length and $R_{3}$ has even length, it must be the case that $C_{r}$ has even length by our assumption. However, this means that all cycles in $C$ have even lengths, contradicting the aperiodicity of $G$.

The case of odd length paths can be proved in the same way.

Proposition A.2. Let $\bar{G}=(V, E, \bar{A})$ be an ergodic unsigned digraph, with transition probability matrix $\bar{P}$ and stationary distribution vector $\pi . \bar{P}^{t}-\mathbf{1} \pi^{T}=\left(\bar{P}-\mathbf{1} \pi^{T}\right)^{t}$ holds for any integer $t>0$.

Proof. Using the facts that $\bar{P} \mathbf{1}=\mathbf{1}$ and $\pi^{T} \bar{P}=\pi^{T}$, it is easy to prove by induction that for any integer $t>0 \bar{P}^{t}-\mathbf{1} \pi^{T}=\left(\bar{P}-\mathbf{1} \pi^{T}\right)^{t}$ holds.

\section{APPENDIX B. SPECIAL MATRIX POWER SERIES}

Proposition B.1. Let $X \in \mathbb{R}^{m \times m}, Y \in \mathbb{R}^{m \times n}$ and $Z \in \mathbb{R}^{n \times n}$. If $\lim _{t \rightarrow \infty} X^{t}=$ $\lim _{t \rightarrow \infty} Z^{t}=\mathbf{0}$, the following equalities hold:

$$
\begin{aligned}
& \text { (i) } \lim _{t \rightarrow \infty} \sum_{i=0}^{t-1} X^{i}=(I-X)^{-1}, \\
& \text { (ii) } \lim _{t \rightarrow \infty} \sum_{i=0}^{t-1} X^{i} Y Z^{t-1-i}=0,
\end{aligned}
$$




\section{Proof.}

(i) Let $\rho(X)$ be the spectral radius of matrix $X$, i.e., the largest absolute value of the eigenvalues of $X$. Notice that $\lim _{t \rightarrow \infty} X^{t}=\mathbf{0}$ if and only if $\rho(X)<1$.

We first claim that $I-X$ and $I-Z$ are invertible. Suppose $I-X$ is not invertible; there is a nonzero vector $p$ such that $(I-X) p=\mathbf{0}$. Therefore, $p$ is the eigenvector of $X$ with eigenvalue 1 , which contradicts $\lim _{t \rightarrow \infty} X^{t}=\mathbf{0}$. The same argument can be applied to $I-Z$. Hence, the left-hand side of (B.1) equals

$$
\lim _{t \rightarrow \infty} \sum_{i=0}^{t} X^{i}=\lim _{t \rightarrow \infty}(I-X)^{-1}\left(I-X^{t+1}\right)=(I-X)^{-1} .
$$

(ii) The max-norm of $X$ is given by $\|X\|_{\max }=\max _{i, j \leq m}\left\{X_{i j}\right\}$. Let $X=Q_{X} \mathcal{J} Q_{X}^{-1}$ be the standard Jordan form of $X$, where $Q_{X}$ is an invertible matrix. Denote $J=\mathbf{1 1}^{T}$ as the all-one matrix. Hence, we have

$$
\begin{aligned}
\left\|X^{i}\right\|_{\max } & =\left\|Q_{X} \mathcal{J}^{i} Q_{X}^{-1}\right\|_{\max } \leq\left\|Q_{X}\right\|_{\max }\left\|Q_{X}^{-1}\right\|_{\max }\left\|J \mathcal{J}^{i} J\right\|_{\max } \\
& \leq\left\|Q_{X}\right\|_{\max }\left\|Q_{X}^{-1}\right\|_{\max } m^{2}\left\|\mathcal{J}^{i}\right\|_{\max }
\end{aligned}
$$

$\mathcal{J}^{i}$ is in form as

$$
\mathcal{J}^{i}=\left[\begin{array}{ccccc}
\lambda_{1}^{i} & C_{i}^{1} \lambda_{1}^{i-1} & C_{i}^{2} \lambda_{1}^{i-2} & 0 & 0 \\
0 & \lambda_{1}^{i} & C_{i}^{1} \lambda_{1}^{i-1} & 0 & 0 \\
0 & 0 & \lambda_{1}^{i} & 0 & 0 \\
0 & 0 & 0 & \lambda_{m_{0}}^{i} & C_{i}^{1} \lambda_{m_{0}}^{i-1} \\
0 & 0 & 0 & 0 & \lambda_{m_{0}}^{i}
\end{array}\right],
$$

where $C_{i}^{\ell}=\frac{i ! \ell !}{(i-\ell) !} \leq i^{m}$ and each nonzero entry in $\mathcal{J}^{i}$ can be expressed as $C_{\ell}^{i} \lambda_{k}^{i-\ell}$, $1 \leq k \leq m_{0}, 1 \leq \ell \leq \ell_{0}(k)$, with $m_{0}$ as the number of different eigenvalues of $X$ and $\ell_{0}(k)$ as the multiplicity of the $k$ th eigenvalue of $X$. Hence, the absolute value of each nonzero entry in $\mathcal{J}^{i}$ is upper bounded as $\left|C_{i}^{\ell} \lambda_{k}^{i-\ell}\right| \leq i^{m} \rho(X)^{i-m}$, which implies that

$$
\left\|X^{i}\right\|_{\max } \leq\left\|Q_{X}\right\|_{\max }\left\|Q_{X}^{-1}\right\|_{\max } m^{2} i^{m} \rho(X)^{i-m}
$$

Let $\rho=\max (\rho(X), \rho(Z))$; we have

$$
\begin{aligned}
& \quad \lim _{t \rightarrow \infty}\left\|\sum_{i=0}^{t-1} X^{i} Y Z^{t-1-i}\right\|_{\max } \leq \lim _{t \rightarrow \infty} t m n\left\|X^{i}\right\|_{\max }\|Y\|_{\max }\left\|Z^{t-1-i}\right\|_{\max } \\
& \leq \lim _{t \rightarrow \infty} t m n T_{\max }\left(m^{2} t^{m} \rho^{i-m}\right)\left(n^{2} t^{n} \rho^{t-i-1-n}\right) \leq \lim _{t \rightarrow \infty} m^{3} n^{3} T_{\max } t^{m+n+1} \rho^{t-1-n-m}=0,
\end{aligned}
$$

where $T_{\max }=\|Y\|_{\max }\left\|Q_{X}\right\|_{\max }\left\|Q_{X}^{-1}\right\|_{\max }\left\|Q_{Z}\right\|_{\max }\left\|Q_{Z}^{-1}\right\|_{\max }$.

\section{APPENDIX C. ILLUSTRATION OF EXPONENTIAL CONVERGENCE TIME OF $P^{t}$ ON ERGODIC DIGRAPH}

Given an unsigned ergodic digraph $\bar{G}=(V, E, \bar{A})$, with transition probability matrix $\bar{P}$, it has fixed stationary distribution $\pi$, i.e., $\pi^{T}=\pi^{T} \bar{P}$. 


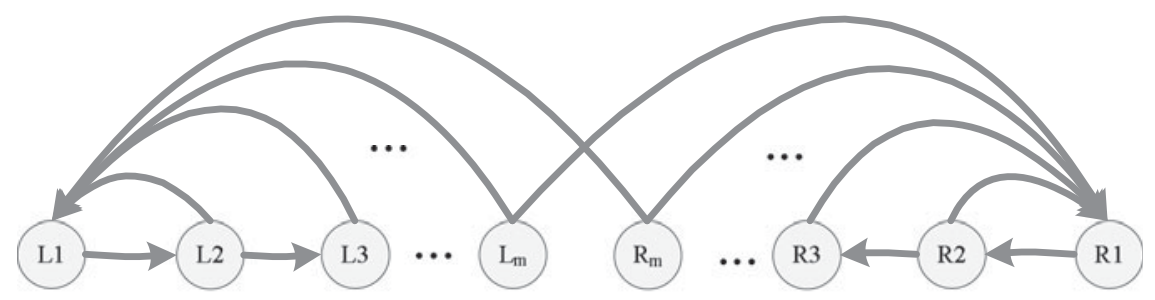

Figure 23 An example digraph with exponential convergence time. All edges are with unit weights.

The convergence time (or mixing time) of a random walk Markov chain on $G$ is the time until the Markov chain is "close" to its stationary distribution $\pi$. To be precise, for an initial distribution $x_{0}$, let $x_{t}^{T}=x_{0}^{T} \bar{P}^{t}$ be the distribution at step $t$. The variation distance mixing time is defined as the smallest $t$ such that for any subset $W \subseteq V$,

$$
\left|\left(x_{t}^{T}-\pi^{T}\right) e_{W}\right| \leq \frac{1}{4}
$$

where $e_{W}$ is the vector such that $e_{W}(i)=1$ if $i \in W$, and $e_{W}(i)=0$ if $i \in V \backslash W$.

The convergence time is said to be exponentially large if there exists $x_{0}$ such that the convergence time of the random walk starting from $x_{0}$ is $2^{\Omega(n)}$, where $n=|V|$. Lemma C.1 illustrates that the convergence time of random walk on ergodic digraphs could be exponentially large.

Lemma C.1. There exist ergodic digraphs such that the convergence times of the random walks on these digraphs are exponentially large.

Proof. We prove this by construction. Figure 23 shows an example digraph $G$, with $|V|=2 m$ nodes. On the left-hand side, there are $m \geq 3$ nodes $L_{1}, L_{2}, \cdots, L_{m}$ connected by $m-1$ directed edges from $L_{1}$ to $L_{m}$, and every node $L_{i}$ with $i>1$ has a directed connection to the leftmost node $L_{1}$. The right-hand side nodes have symmetric connections as the left-hand side. Moreover, nodes $L_{m}$ and $R_{m}$ also have one more connection to $R_{1}$ and $L_{1}$, respectively, which connect two components together. It is clear that the graph is strongly connected and aperiodic (there exist cycles of lengths 2 and 3), and, thus, is ergodic.

Let $x_{t}\left(L_{i}\right)$ denote the probability that the random walk is at node $L_{i}$ at step $t$, and $x\left(L_{i}\right)$ be its stationary distribution. Similarly define $x_{t}\left(R_{i}\right)$ and $x\left(R_{i}\right)$ for node $R_{i}$. The graph is symmetric, thus, we have $x\left(L_{i}\right)=x\left(R_{i}\right)$ for $1 \leq i \leq m$. Let $x\left(L_{1}\right)=x\left(R_{1}\right)=\rho / 4$, we have $x\left(L_{i}\right)=x\left(R_{i}\right)=\rho / 2^{i}$ for $i=2,3, \ldots, m$. Then, by solving $\sum_{i=1}^{m}\left(x\left(L_{i}\right)+x\left(R_{i}\right)\right)=1$, we obtain $\rho=\frac{2^{m-1}}{3 \cdot 2^{m-2}-1}$. It is easy to verify that indeed the obtained $x$ is the stationary distribution of the random walks on the digraph.

Then, we consider the initial distribution as $x_{0}=[1,0,0, \ldots, 0]$, and the subset $W=\left\{R_{1}, \cdots, R_{m}\right\}$ including all $m$ nodes on the right-hand side. Let $x_{t}(W)=x_{t}^{T} \cdot e_{W}$ denote the total probability that the random walk is in some node in $W$ at step $t$. The only edge from the left half to the right half is the edge from $L_{m}$ to $R_{1}$. Thus, all additions to $x_{t+1}(W)$ from $x_{t}(W)$ come from this edge, namely, $x_{t+1}(W)-x_{t}(W) \leq x_{t}\left(L_{m}\right) / 2$. We now bound $x_{t}\left(L_{m}\right)$. For $t \leq m-1$, we know that $x_{t}\left(L_{m}\right)=0$. For $t \geq m$, we have

$$
x_{t}\left(L_{m}\right)=x_{t-1}\left(L_{m-1}\right) / 2=x_{t-2}\left(L_{m-2}\right) / 2^{2}=\cdots=x_{t-m+2}\left(L_{2}\right) / 2^{m-2} \leq 1 / 2^{m-2} .
$$


Hence, we have

$$
x_{t}(W)=\sum_{i=1}^{t}\left(x_{i}(W)-x_{i-1}(W)\right) \leq t \cdot x_{t}\left(L_{m}\right) / 2 \leq t / 2^{m-1} .
$$

Therefore, the smallest $t$ that satisfies $\left|\left(x_{t}^{T}-\pi^{T}\right) e_{W}\right|=\left|x_{t}(W)-1 / 2\right| \leq 1 / 4$ is such that $x_{t}(W) \geq 1 / 4$, which implies that $t / 2^{m-1} \geq 1 / 4$ and $t \geq 2^{m-3}$. This completes the proof.

\section{REFERENCES}

[1] M. Ángeles Serrano, K. Klemm, F. Vazquez, V. Eguíuuz, and M. San Miguel. "Conservation Laws for Voter-Like Models on Random Directed Networks." Journal of Statistical Mechanics: Theory and Experiment 2009:10(2009), 1-18.

[2] S. Bharathi, D. Kempe, and M. Salek. "Competitive Influence Maximization in Social Networks.” In WINE, 306-311. Berlin, Heidelberg: Springer, 2007.

[3] C. Borgs, J. Chayes, A. Kalai, A. Malekian, and M. Tennenholtz. "A Novel Approach to Propagating Distrust.” In WINE, 2010. Berlin, Heidelberg: Springer, 2010.

[4] A. Borodin, Y. Filmus, and J. Oren. "Threshold Models for Competitive Influence in Social Networks." In WINE, 2010. Berlin, Heidelberg: Springer, 2010.

[5] C. Budak, D. Agrawal, and A. E. Abbadi. "Limiting the Spread of Misinformation in Social Networks." In $W W W, 2011$. New York, NY: ACM, 2011.

[6] W. Chen, A. Collins, R. Cummings, T. Ke, Z. Liu, D. Rincón, X. Sun, Y. Wang, W. Wei, and Y. Yuan. "Influence Maximization in Social Networks When Negative Opinions May Emerge and Propagate." In SDM, 2011. Mesa, AZ: SIAM, 2011.

[7] W. Chen, C. Wang, and Y. Wang. "Scalable Influence Maximization for Prevalent Viral Marketing in Large-Scale Social Networks." In KDD, 2010. New York, NY: ACM, 2010.

[8] W. Chen, Y. Wang, and S. Yang. "Efficient Influence Maximization in Social Networks." In KDD, 2009. New York, NY: ACM, 2009.

[9] W. Chen, Y. Yuan, and L. Zhang. "Scalable Influence Maximization in Social Networks Under the Linear Threshold Model.” In ICDM, 2010. IEEE, 2010.

[10] K. Chiang, N. Natarajan, A. Tewari, and I. Dhillon. "Exploiting Longer Cycles for Link Prediction in Signed Networks." In CIKM, 2011. New York, NY: ACM, 2011.

[11] F. R. K. Chung. "Laplacians and the Cheeger Inequality for Directed Graphs." Annals of Combinatorics 9(2005), 1-19.

[12] F. Chung and A. Tsiatas. "Hypergraph Coloring Games and Voter Models." Internet Mathematics 10:1-2(2012), 66-68.

[13] P. Clifford and A. Sudbury. "A Model for Spatial Conflict.” Biometrika, 60:3(1973), 581.

[14] D. Easley and J. Kleinberg. Networks, Crowds, and Markets: Reasoning About a Highly Connected World. Cambridge, UK: Cambridge University Press, 2010.

[15] Epinions. Dataset. http://www.epinions.com/.

[16] E. Even-Dar and A. Shapira. "A Note on Maximizing the Spread of Influence in Social Networks." In WINE, 2007. Berlin, Heidelberg: Springer, 2007.

[17] A. Goyal, F. Bonchi, and L. V. S. Lakshmanan. "A Data-Based Approach to Social Influence Maximization." PVLDB 5:1(2008),73-84.

[18] A. Goyal, W. Lu, and L. V. S. Lakshmanan. "Simpath: An Efficient Algorithm for Influence Maximization Under the Linear Threshold Model.” In ICDM, 2011. IEEE, 2011.

[19] X. He, G. Song, W. Chen, and Q. Jiang. "Influence Blocking Maximization in Social Networks Under the Competitive Linear Threshold Model." In SDM, 2012. Anaheim, CA: SIAM, 2012.

[20] R. Holley and T. Liggett. "Ergodic Theorems for Weakly Interacting Infinite Systems and the Voter Model." The Annals of Probability 3:4(1975), 643-663. 
[21] D. Kempe, J. Kleinberg, and E. Tardos. "Maximizing the Spread of Influence Through a Social Network." In KDD, 2003. New York, NY: ACM, 2003.

[22] M. Kimura and K. Saito. "Tractable Models for Information Diffusion in Social Networks." In PKDD, 2006. Berlin, Heidelberg: Springer, 2006.

[23] J. Kunegis, S. Schmidt, A. Lommatzsch, J. Lerner, E.W. D. Luca, and S. Albayrak. "Spectral Analysis of Signed Graphs for Clustering, Prediction and Visualization.” In SDM, 2010. Columbus, OH: SIAM, 2010.

[24] J. Leskovec, D. Huttenlocher, and J. Kleinberg. "Predicting Positive and Negative Links in Online Social Networks." In WWW, 2010. New York, NY: ACM, 2010.

[25] J. Leskovec, D. Huttenlocher, and J. Kleinberg. "Signed Networks in Social Media." In CHI. New York, NY: ACM, 2010.

[26] J. Leskovec, A. Krause, C. Guestrin, C. Faloutsos, J. M. VanBriesen, and N. S. Glance. "CostEffective Outbreak Detection in Networks." In KDD, 2007. New York, NY: ACM, 2007.

[27] Y. Li, W. Chen, Y. Wang, and Z.-L. Zhang. "Influence Diffusion Dynamics and Influence Maximization in Social Networks with Friend and Foe Relationships." In WSDM '13: Proceedings of the 6th ACM International Conference on Web Search and Data Mining. New York, NY: ACM, 2013.

[28] Y. Li, Z. Zhang, and J. Bao. "Mutual or Unrequited Love: Identifying Stable Clusters in Social Networks with Uni-and Bi-Directional Links." WAW '12: Proceedings of the 9th Workshop on Algorithms and Models for the Web Graph, pp. 113-125, Springer, 2012.

[29] Y. Li and Z.-L. Zhang. "Random Walks on Digraphs: A Theoretical Framework for Estimating Transmission Costs in Wireless Routing. In INFOCOM, 2010. IEEE, 2010.

[30] Y. Li and Z.-L. Zhang. "Random Walks on Digraphs, the Generalized Digraph Laplacian and the Degree of Asymmetry." In LNCS WAW 2010: Proceedings of the 7th Workshop on Algorithms and Models for the Web Graph, 2010. Berlin: Springer, 2010.

[31] Y. Li and Z.-L. Zhang. "Digraph Laplacian and the Degree of Asymmetry." Internet Mathematics, 8:4(2012), 381-401.

[32] Y. Li and Z.-L. Zhang. "Random Walks and Green's Function on Digraphs: A Framework for Estimating Wireless Transmission Costs. IEEE/ACM Transactions on Networking, PP(99):1(2012),1-14.

[33] Y. Li, Z.-L. Zhang, and D. Boley. "The Routing Continuum from Shortest-Path to All-Path: A Unifying Theory." In ICDCS'11: 31st International Conference on Distributed Computing Systems, pp. 847-856. IEEE, 2011.

[34] Y. Li, Z.-L. Zhang, and D. Boley. "From Shortest-Path to All-Path: The Routing Continuum Theory and Its Applications." IEEE Transactions on Parallel and Distributed Systems, PP(99):1(2014),1-11.

[35] H. Ma, H. Yang, M. R. Lyu, and I. King. "Mining Social Networks Using Heat Diffusion Processes for Marketing Candidates Selection." In CIKM, 2008. New York, NY: ACM, 2008.

[36] N. Masuda and H. Ohtsuki. "Evolutionary Dynamics and Fixation Probabilities in Directed Networks." New Journal of Physics 11:033012(2009), 1-25.

[37] R. Narayanam and Y. Narahari. "Determining the Top-k Nodes in Social Networks Using the Shapley Value." In AAMAS, 2008. ACM, 2008.

[38] N. Pathak, A. Banerjee, and J. Srivastava. "A Generalized Linear Threshold Model for Multiple Cascades.” In ICDM, 2010. IEEE, 2010. [Slashdot n.d.] Slashdot. Dataset. http://slashdot.org/.

[39] Slashdot. Dataset. http://slashdot.org/.

[40] V. Sood, T. Antal, and S. Redner. "Voter Models on Heterogeneous Networks." Physical Review E 77:4(2008), 041121.

[41] W. Stewart. "Numerical Methods for Computing Stationary Distributions of Finite Irreducible Markov Chains." Computational Probability. New York, NY: Springer US, 2000. 